\title{
A comprehensive classification system for lipids ${ }^{1}$
}

\author{
Eoin Fahy, $*$ Shankar Subramaniam, ${ }^{\dagger}$ H. Alex Brown, $₫$ Christopher K. Glass, ** \\ Alfred H. Merrill, Jr., ${ }^{\dagger \dagger}$ Robert C. Murphy, $\$ \S$ Christian R. H. Raetz, $* * *$ David W. Russell, ${ }^{\dagger \dagger}$ \\ Yousuke Seyama, $\$ \S \S$ Walter Shaw, $* * * *$ Takao Shimizu, ${ }^{\dagger \dagger}+$ Friedrich Spener, $\$ \S \S \S$ \\ Gerrit van Meer, ${ }^{* * * * *}$ Michael S. VanNieuwenhze, ${ }^{\dagger+}+\dagger \dagger$ Stephen H. White,,$\S \S \S \S$ \\ Joseph L. Witztum, $* * * * * *$ and Edward A. Dennis $2,+\dagger+\dagger \dagger$ \\ San Diego Supercomputer Center,* University of California, San Diego, 9500 Gilman Drive, La Jolla, CA \\ 92093-0505; Department of Bioengineering, ${ }^{\dagger}$ University of California, San Diego, 9500 Gilman Drive, La Jolla, \\ CA 92093-0412; Department of Pharmacology, $\$$ Vanderbilt University Medical Center, Nashville, TN 37232- \\ 6600; Department of Cellular and Molecular Medicine, ** University of California, San Diego, 9500 Gilman \\ Drive, La Jolla, CA 92093-0651; School of Biology, ${ }^{\dagger}$ Georgia Institute of Technology, Atlanta, GA 30332-0230; \\ Department of Pharmacology, \$§ University of Colorado Health Sciences Center, Aurora, CO 80045-0508; \\ Department of Biochemistry, ${ }^{* *}$ Duke University Medical Center, Durham, NC 27710; Department of \\ Molecular Genetics, ${ }^{+\dagger}$ University of Texas Southwestern Medical Center, Dallas, TX 75390-9046; Faculty of \\ Human Life and Environmental Sciences, ${ }^{\$ \S}$ Ochanomizu University, Tokyo 112-8610, Japan; Avanti Polar \\ Lipids, Inc., ${ }^{* * * *}$ Alabaster, AL 35007; Department of Biochemistry and Molecular Biology, ${ }^{\dagger+\dagger}$ Faculty of \\ Medicine, University of Tokyo, Tokyo 113-0033, Japan; Department of Molecular Biosciences, $\$ \$ \S \S$ University of \\ Graz, 8010 Graz, Austria; Department of Membrane Enzymology,***** Institute of Biomembranes, Utrecht \\ University, 3584 CH Utrecht, The Netherlands; Department of Chemistry and Biochemistry, ${ }^{\dagger+\dagger \dagger \dagger}$ University of \\ California, San Diego, 9500 Gilman Drive, La Jolla, CA 92093-0358; Department of Physiology and \\ Biophysics, $\$ \S \S \S \S$ University of California at Irvine, Irvine, CA 92697-4560; Department of Medicine,****** \\ University of California, San Diego, 9500 Gilman Drive, La Jolla, CA 92093-0682; and Department of \\ Chemistry and Biochemistry and Department of Pharmacology, ${ }^{+1+\dagger+\dagger}$ University of California, San Diego, \\ La Jolla, CA 92093-0601
}

\begin{abstract}
Lipids are produced, transported, and recognized by the concerted actions of numerous enzymes, binding proteins, and receptors. A comprehensive analysis of lipid molecules, "lipidomics," in the context of genomics and proteomics is crucial to understanding cellular physiology and pathology; consequently, lipid biology has become a major research target of the postgenomic revolution and systems biology. To facilitate international communication about lipids, a comprehensive classification of lipids with a common platform that is compatible with informatics requirements has been developed to deal with the massive amounts of data that will be generated by our lipid community. As an initial step in this development, we divide lipids into eight categories (fatty acyls, glycerolipids, glycerophospholipids, sphingolipids, sterol lipids, prenol lipids, saccharolipids, and polyketides) containing distinct classes and subclasses of molecules, devise a common manner of representing the chemical structures of individual lipids and their derivatives, and provide a 12 digit identifier for each unique lipid molecule. The lipid classification scheme is chemically based and driven by the distinct hydrophobic and hydrophilic elements that compose the lipid. This structured vocabulary will facilitate the systematization of lipid biology and enable the cataloging of lipids and their properties in a way that is compatible with
\end{abstract}

Manuscript received 22 December 2004 and in revised form 4 February 2005.

DOI 10.1194/jlr.E400004-JLR200
Published, JLR Papers in Press, February 16, 2005.

other macromolecular databases.-Fahy, E., S. Subramaniam, H. A. Brown, C. K. Glass, A. H. Merrill, Jr., R. C. Murphy, C. R. H. Raetz, D. W. Russell, Y. Seyama, W. Shaw, T. Shimizu, F. Spener, G. van Meer, M. S. VanNieuwenhze, S. H. White, J. L. Witztum, and E. A. Dennis. A comprehensive classification system for lipids. J. Lipid Res. 2005. 46: 839-861.

Supplementary key words lipidomics $\bullet$ informatics $\bullet$ nomenclature $\bullet$ chemical representation $\bullet$ fatty acyls $\bullet$ glycerolipids $\bullet$ glycerophospholipids • sphingolipids • sterol lipids • prenol lipids • saccharolipids • polyketides

The goal of collecting data on lipids using a "systems biology" approach to lipidomics requires the development of a comprehensive classification, nomenclature, and chemical representation system to accommodate the myriad lipids that exist in nature. Lipids have been loosely defined as biological substances that are generally hydrophobic in nature and in many cases soluble in organic solvents (1). These chemical properties cover a broad range of mole-

\footnotetext{
${ }^{1}$ The evaluation of this manuscript was handled by the former Editor-in-Chief Trudy Forte.

${ }^{2}$ To whom correspondence should be addressed.

e-mail: edennis@ucsd.edu
}

Copyright $(2005$ by the American Society for Biochemistry and Molecular Biology, Inc. This article is available online at http://www.jlr.org 
cules, such as fatty acids, phospholipids, sterols, sphingolipids, terpenes, and others (2). The LIPID MAPS (LIPID Metabolites And Pathways Strategy; http://www.lipidmaps.org), Lipid Library (http:/ /lipidlibrary.co.uk), Lipid Bank (http:/ / lipidbank.jp), LIPIDAT (http://www.lipidat.chemistry.ohiostate.edu), and Cyberlipids (http://www.cyberlipid.org) websites provide useful online resources for an overview of these molecules and their structures. More accurate definitions are possible when lipids are considered from a structural and biosynthetic perspective, and many different classification schemes have been used over the years. However, for the purpose of comprehensive classification, we define lipids as hydrophobic or amphipathic small molecules that may originate entirely or in part by carbanion-based condensations of thioesters (fatty acids, polyketides, etc.) and/or by carbocation-based condensations of isoprene units (prenols, sterols, etc.). Additionally, lipids have been broadly subdivided into "simple" and "complex" groups, with simple lipids being those yielding at most two types of products on hydrolysis (e.g., fatty acids, sterols, and acylglycerols) and complex lipids (e.g., glycerophospholipids and glycosphingolipids) yielding three or more products on hydrolysis. The classification scheme presented here organizes lipids into well-defined categories that cover eukaryotic and prokaryotic sources and that is equally applicable to archaea and synthetic (manmade) lipids.

Lipids may be categorized based on their chemically functional backbone as polyketides, acylglycerols, sphingolipids, prenols, or saccharolipids. However, for historical and bioinformatics advantages, we chose to separate fatty acyls from other polyketides, the glycerophospholipids from the other glycerolipids, and sterol lipids from other prenols, resulting in a total of eight primary categories. An important aspect of this scheme is that it allows for subdivision of the main categories into classes and subclasses to handle the existing and emerging arrays of lipid structures. Although any classification scheme is in part subjective as a result of the structural and biosynthetic complexity of lipids, it is an essential prerequisite for the organization of lipid research and the development of systematic methods of data management. The classification scheme presented here is chemically based and driven by the distinct hydrophobic and hydrophilic elements that constitute the lipid. Biosynthetically related compounds that are not technically lipids because of their water solubility are included for completeness in this classification scheme.

The proposed lipid categories listed in Table $\mathbf{1}$ have names that are, for the most part, well accepted in the literature. The fatty acyls (FA) are a diverse group of molecules synthesized by chain elongation of an acetyl-CoA primer with malonyl-CoA (or methylmalonyl-CoA) groups that may contain a cyclic functionality and/or are substituted with heteroatoms. Structures with a glycerol group are represented by two distinct categories: the glycerolipids (GL), which include acylglycerols but also encompass alkyl and 1Z-alkenyl variants, and the glycerophospholipids (GP), which are defined by the presence of a phosphate (or phosphonate) group esterified to one of the glycerol hydroxyl groups. The sterol lipids (ST) and prenol lipids (PR) share a common biosynthetic pathway via the polymerization of dimethylallyl pyrophosphate/isopentenyl pyrophosphate but have obvious differences in terms of their eventual structure and function. Another well-defined category is the sphingolipids (SP), which contain a longchain base as their core structure. This classification does not have a glycolipids category per se but rather places glycosylated lipids in appropriate categories based on the identity of their core lipids. It also was necessary to define a category with the term "saccharolipids" (SL) to account for lipids in which fatty acyl groups are linked directly to a sugar backbone. This SL group is distinct from the term "glycolipid" that was defined by the International Union of Pure and Applied Chemists (IUPAC) as a lipid in which the fatty acyl portion of the molecule is present in a glycosidic linkage. The final category is the polyketides (PK), which are a diverse group of metabolites from plant and microbial sources. Protein modification by lipids (e.g., fatty acyl, prenyl, cholesterol) occurs in nature; however, these proteins are not included in this database but are listed in protein databases such as GenBank (http://www.ncbi.nlm. nih.gov) and SwissProt (http://www.ebi.ac.uk/swissprot/).

\section{LIPID NOMENCLATURE}

A naming scheme must unambiguously define a lipid structure in a manner that is amenable to chemists, biologists, and biomedical researchers. The issue of lipid nomenclature was last addressed in detail by the International Union of Pure and Applied Chemists and the International Union of Biochemistry and Molecular Biology (IUPAC-IUBMB) Commission on Biochemical Nomenclature in 1976, which subsequently published its recommendations (3). Since then, a number of additional documents relating to the naming of glycolipids (4), prenols (5), and steroids (6) have been released by this commission and placed on the IUPAC website (http://www. chem.qmul.ac.uk/iupac/). A large number of novel lipid classes have been discovered during the last three decades that have not yet been systematically named. The present classification includes these new lipids and incorporates a consistent nomenclature.

In conjunction with our proposed classification scheme, we provide examples of systematic (or semisystematic) names for the various classes and subclasses of lipids. The nomenclature proposal follows existing IUPAC-IUBMB rules closely and should not be viewed as a competing format. The main differences involve $a$ ) clarification of the use of core structures to simplify systematic naming of some of the more complex lipids, and $b$ ) provision of systematic names for recently discovered lipid classes.

Key features of our lipid nomenclature scheme are as follows:

a) The use of the stereospecific numbering $(s n)$ method to describe glycerolipids and glycerophospholipids (3). The glycerol group is typically acylated or alkylated at the $s n-1$ and/or $s n-2$ position, with the exception of some lipids 
TABLE 1. Lipid categories and examples

\begin{tabular}{lcl}
\hline Category & Abbreviation & \multicolumn{1}{c}{ Example } \\
\hline Fatty acyls & FA & dodecanoic acid \\
Glycerolipids & GL & 1-hexadecanoyl-2-(9Z-octadecenoyl)-sn-glycerol \\
Glycerophospholipids & GP & 1-hexadecanoyl-2-(9Z-octadecenoyl)-sn-glycero-3-phosphocholine \\
Sphingolipids & SP & $N$-(tetradecanoyl)-sphing-4-enine \\
Sterol lipids & ST & cholest-5-en-3ß-ol \\
Prenol lipids & PR & $2 E, 6 E$-farnesol \\
Saccharolipids & SL & UDP-3- $O$ - $(3 R$-hydroxy-tetradecanoyl)- $\alpha \mathrm{D}$ - $N$-acetylglucosamine \\
Polyketides & PK & aflatoxin $\mathrm{B}_{1}$ \\
\hline
\end{tabular}

that contain more than one glycerol group and archaebacterial lipids in which $s n-2$ and/or $s n-3$ modification occurs.

b) Definition of sphinganine and sphing-4-enine as core structures for the sphingolipid category, where the D-erythro or $2 S, 3 R$ configuration and $4 E$ geometry (in the case of sphing-4-enine) are implied. In molecules containing stereochemistries other than the $2 S, 3 R$ configuration, the full systematic names are to be used instead (e.g., $2 R$-amino1,3R-octadecanediol).

c) The use of core names such as cholestane, androstane, and estrane for sterols.

d) Adherence to the names for fatty acids and acyl chains (formyl, acetyl, propionyl, butyryl, etc.) defined in Appendices $\mathrm{A}$ and $\mathrm{B}$ of the IUPAC-IUBMB recommendations (3).

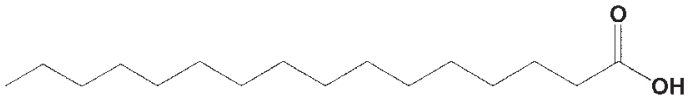

(a) Fatty Acyls: hexadecanoic acid

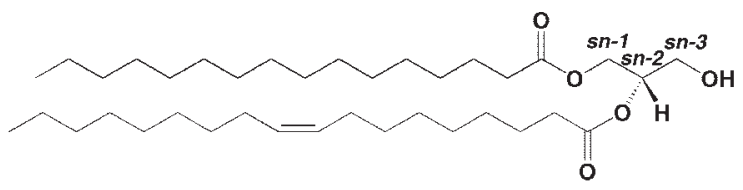

(b) Glycerolipids: 1-hexadecanoyl-2-(9Z-octadecenoyl)-sn-glycerol

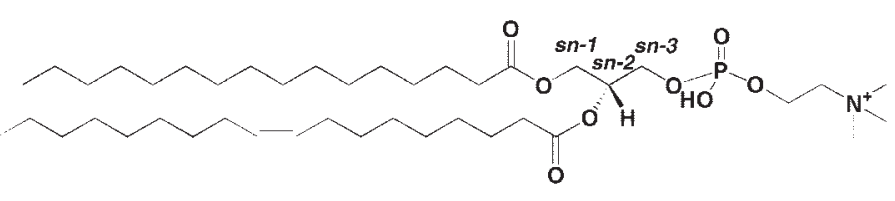

(c) Glycerophospholipids: 1-hexadecanoyl-2-

(9Z-octadecenoyl)-sn-glycero-3-phosphocholine

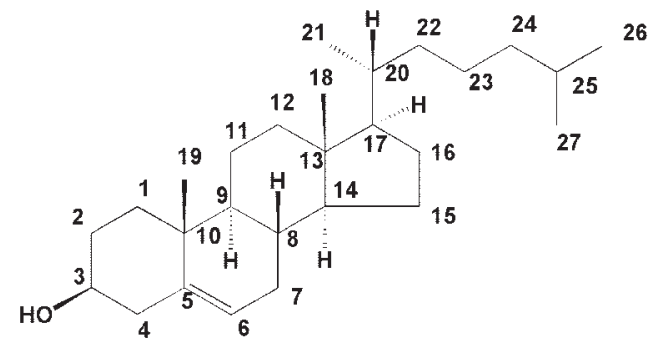

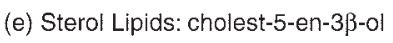

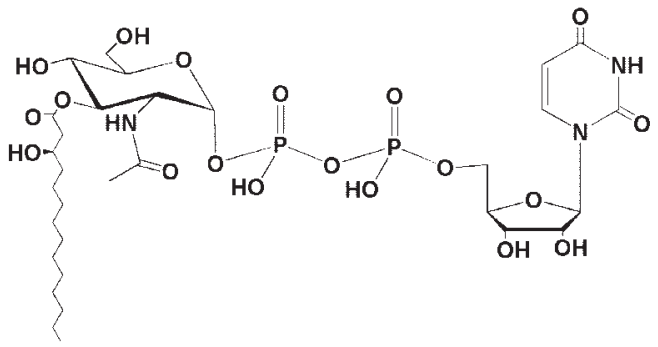

(g) Saccharolipids: UDP-3-O-(3R-hydroxy -tetradecanoyl)- $\alpha \mathrm{D}-\mathrm{N}$-acetylglucosamine

Fig. 1. Representative structures for each lipid category.

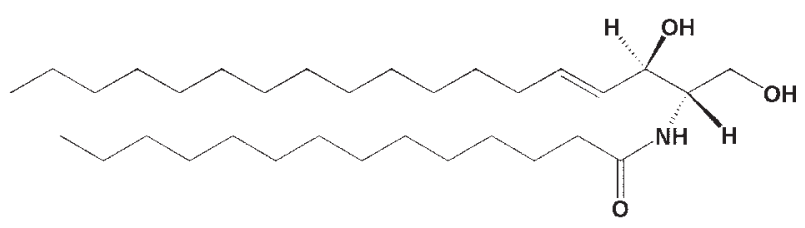

(d) Sphingolipids: $\mathrm{N}$-(tetradecanoyl)-sphing-4-enine

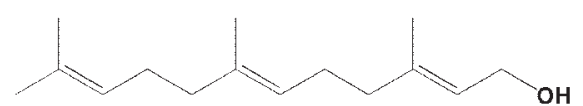

(f) Prenol Lipids: 2E,6E-farnesol

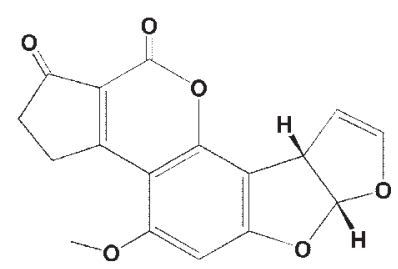

(h) Polyketides: aflatoxin B1 
TABLE 2. Format of 12 character LIPID ID

\begin{tabular}{llc}
\hline Characters & \multicolumn{1}{c}{ Description } & Example \\
\hline $1-2$ & Fixed database designation & LM \\
$3-4$ & Two letter category code & FA \\
$5-6$ & Two digit class code & 03 \\
$7-8$ & Two digit subclass code & 02 \\
$9-12$ & Unique four character identifier within subclass & 7312 \\
\hline
\end{tabular}

e) The adoption of a condensed text nomenclature for the glycan portions of lipids, where sugar residues are represented by standard IUPAC abbreviations and where the anomeric carbon locants and stereochemistry are included but the parentheses are omitted. This system has also been proposed by the Consortium for Functional Glycomics (http://web.mit.edu/glycomics/consortium/main.shtml).

$f$ ) The use of $E / Z$ designations (as opposed to trans/cis) to define double bond geometry.

$g$ ) The use of $R / S$ designations (as opposed to $\alpha / \beta$ or $\mathrm{D} / \mathrm{L}$ ) to define stereochemistries. The exceptions are those describing substituents on glycerol $(s n)$ and sterol core structures and anomeric carbons on sugar residues. In these latter special cases, the $\alpha / \beta$ format is firmly established.

h) The common term "lyso," denoting the position lacking a radyl group in glycerolipids and glycerophospholipids, will not be used in systematic names but will be included as a synonym.

i) The proposal for a single nomenclature scheme to cover the prostaglandins, isoprostanes, neuroprostanes, and related compounds, where the carbons participating in the cyclopentane ring closure are defined and where a consistent chain-numbering scheme is used. j) The "d" and "t" designations used in shorthand notation of sphingolipids refer to 1,3-dihydroxy and 1,3,4-trihydroxy long-chain bases, respectively.

\section{LIPID STRUCTURE REPRESENTATION}

In addition to having rules for lipid classification and nomenclature, it is important to establish clear guidelines for drawing lipid structures. Large and complex lipids are difficult to draw, which leads to the use of shorthand and unique formats that often generate more confusion than clarity among lipidologists. We propose a more consistent format for representing lipid structures in which, in the simplest case of the fatty acid derivatives, the acid group (or equivalent) is drawn on the right and the hydrophobic hydrocarbon chain is on the left (Fig. 1). Notable exceptions are found in the eicosanoid class, in which the hydrocarbon chain wraps around in a counterclockwise direction to produce a more condensed structure. Similarly, with regard to the glycerolipids and glycerophospholipids, the radyl chains are drawn with the hydrocarbon chains to the left and the glycerol group depicted horizontally with stereochemistry at the $s n$ carbons defined (if known). The general term "radyl" is used to denote either acyl, alkyl, or 1-alkenyl substituents (http://www.chem.qmul.ac.uk/iupac/lipid/lip1n2. html), allowing for coverage of alkyl and 1 Z-alkenylglycerols. The sphingolipids, although they do not contain a glycerol group, have a similar structural relationship to the glycerophospholipids in many cases and may be drawn with the C1 hydroxyl group of the long-chain base to the right and the alkyl portion to the left. This methodology places the head groups of both sphingolipids and glycerophospho-

TABLE 3. Shorthand notation for selected lipid categories

\begin{tabular}{|c|c|c|c|}
\hline Category & Abbreviation & Class or Subclass & Example $^{a}$ \\
\hline GP & GPCho & Glycerophosphocholines & GPCho $(16: 0 / 9 Z, 12 Z-18: 2)$ \\
\hline GP & GPnCho & Glycerophosphonocholines & \\
\hline GP & GPEtn & Glycerophosphoethanolamines & \\
\hline GP & GPnEtn & Glycerophosphonoethanolamines & \\
\hline GP & GPSer & Glycerophosphoserines & \\
\hline GP & GPGro & Glycerophosphoglycerols & \\
\hline GP & GPGroP & Glycerophosphoglycerophosphates & \\
\hline GP & GPIns & Glycerophosphoinositols & \\
\hline GP & GPInsP & Glycerophosphoinositol monophosphates & \\
\hline GP & GPInsP $_{2}$ & Glycerophosphoinositol bis-phosphates & \\
\hline GP & $\mathrm{GPInsP}_{3}$ & Glycerophosphoinositol tris-phosphates & \\
\hline GP & GPA & Glycerophosphates & \\
\hline GP & GPP & Glyceropyrophosphates & \\
\hline GP & CL & Glycerophosphoglycerophosphoglycerols & \\
\hline GP & CDP-DG & CDP-glycerols & \\
\hline GP & [glycan]GP & Glycerophosphoglucose lipids & \\
\hline GP & [glycan] GPIns & Glycerophosphoinositolglycans & EtN-P-6Man $\alpha 1-2$ Man $\alpha 1-6$ Man $\alpha 1-4 G l c N \alpha 1-6$ GPIns (14:0/14:0) \\
\hline SP & Cer & Ceramides & Cer $(\mathrm{d} 18: 1 / 9 E-16: 1)$ \\
\hline SP & SM & Phosphosphingolipids & $\mathrm{SM}(\mathrm{d} 18: 1 / 24: 0)$ \\
\hline SP & [glycan]Cer & Glycosphingolipids & NeuAc $\alpha 2-3 \mathrm{Gal} \beta 1-4 \mathrm{Glc} \beta-\mathrm{Cer}(\mathrm{d} 18: 1 / 16: 0)$ \\
\hline GL & MG & Monoradyl glycerols & MG $(16: 0 / 0: 0 / 0: 0)$ \\
\hline GL & DG & Diradyl glycerols & DG $(18: 0 / 16: 0 / 0: 0)$ \\
\hline GL & TG & Triradyl glycerols & TG (12:0/14:0/18:0) \\
\hline
\end{tabular}

${ }^{a}$ Shorthand notation for radyl substituents in categories GP and GL are presented in the order of $s n-1$ to $s n-3$. Shorthand notation for category SP is presented in the order of long-chain base and $N$-acyl substituent. Numbers separated by colons refer to carbon chain length and number of double bonds, respectively. 
TABLE 4. Fatty acyls [FA] classes and subclasses

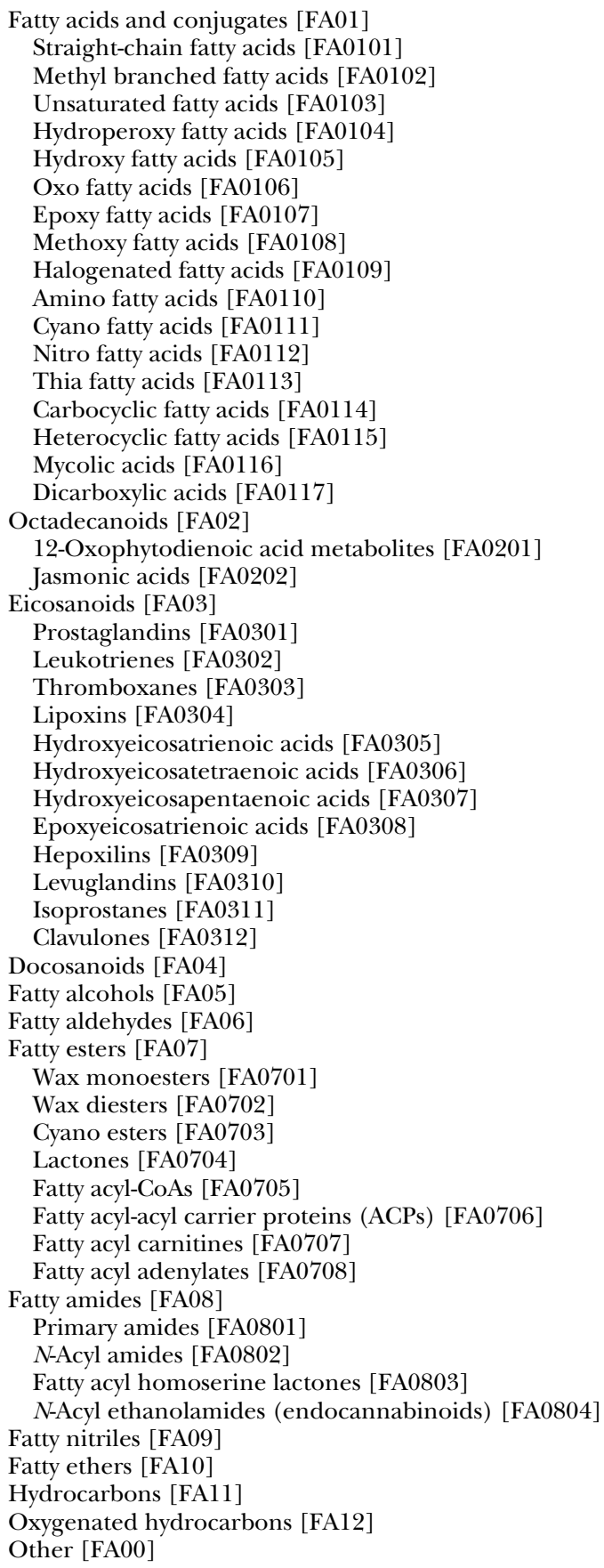

lipids on the right side. Although the structures of sterols do not conform to these general rules of representation, the sterol esters may conveniently be drawn with the acyl group oriented according to these guidelines. In addition, the linear prenols or isoprenoids are drawn in a manner analogous to the fatty acids, with the terminal functional group on the right side. Inevitably, a number of structurally complex lipids, such as acylaminosugar glycans, polycyclic isoprenoids, and polyketides, do not lend themselves to these simplified drawing rules. Nevertheless, we believe that the adoption of the guidelines proposed here will unify chemical representation and make it more comprehensible.
TABLE 5. Glycerolipids [GL] classes and subclasses

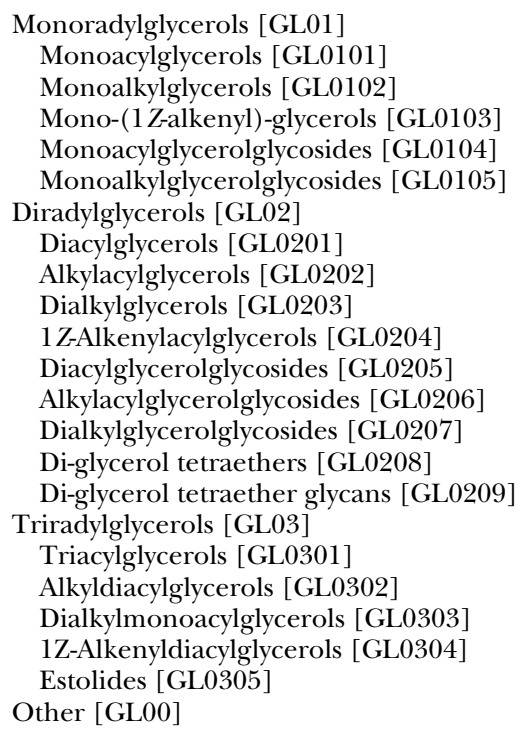

\section{DATABASING LIPIDS, ANNOTATION, AND FUNCTION}

A number of repositories, such as GenBank, SwissProt, and ENSEMBL (http://www.ensembl.org), support nucleic acid and protein databases; however, there are only a few specialized databases [e.g., LIPIDAT (7) and Lipid Bank (8)] that provide a catalog, annotation, and functional classification of lipids. Given the importance of these molecules in cellular function and pathology, there is an imminent need for the creation of a well-organized database of lipids. The first step toward this goal is the establishment of an ontology of lipids that is extensible, flexible, and scalable. Before establishing an ontology, a structured vocabulary is needed, and the IUPAC nomenclature of the 1970s was an initial step in this direction.

The ontology of lipids must contain definitions, meanings, and interrelationships of all objects stored in the database. This ontology is then transformed into a welldefined schema that forms the foundation for a relational database of lipids. The LIPID MAPS project is building a robust database of lipids based on the proposed ontology.

Our database will provide structural and functional annotations and have links to relevant protein and gene data. In addition, a universal data format (XML) will be provided to facilitate exportation of the data into other repositories. This database will enable the storage of curated information on lipids in a web-accessible format and will provide a community standard for lipids.

An important database field will be the LIPID ID, a unique 12 character identifier based on the classification scheme described here. The format of the LIPID ID, outlined in Table 2, provides a systematic means of assigning unique IDs to lipid molecules and allows for the addition of large numbers of new categories, classes, and subclasses in the future, because a maximum of 100 classes /subclasses (00 to 99) may be specified. The last four characters of the 


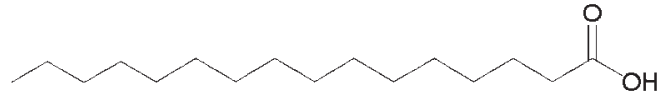

(a) Straight chain fatty acids: hexadecanoic acid

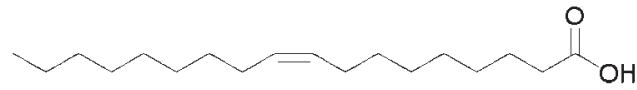

(c) Unsaturated fatty acids: 9Z-octadecenoic acid

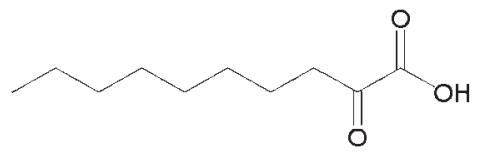

(e) Oxo fatty acids: 2-oxo-decanoic acid

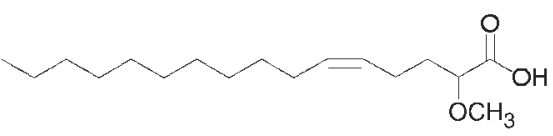

(g) Methoxy fatty acids: 2-methoxy-5Z-hexadecenoic acid

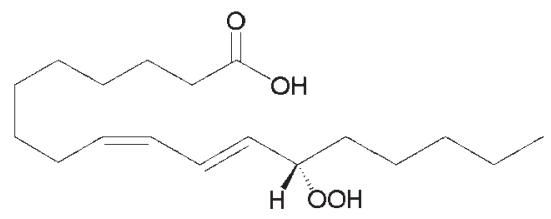

(i) Hydroperoxy fatty acids: 13S-hydroperoxy-

9Z,11E-octadecadienoic acid

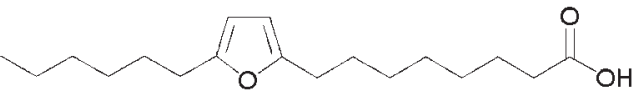

(k) Heterocyclic fatty acids: 8-(5-hexyl-furan-2-yl)-octanoic acid

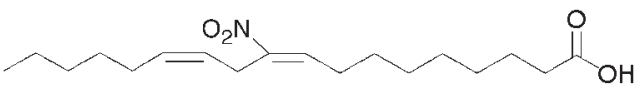

(m) Nitro fatty acids: 10-nitro,9Z,12Z-octadecadienoic acid

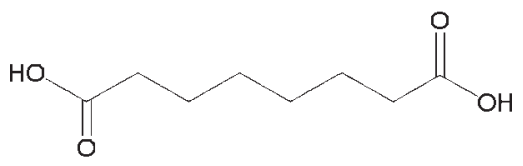

(o) Dicarboxylic acids: 1,8-octanedioic acid

Fig. 2. Representative structures for fatty acyls.

ID constitute a unique identifier within a particular subclass and are randomly assigned. By initially using numeric characters, this allows 9,999 unique IDs per subclass, but with the additional use of 26 uppercase alphabetic characters, a total of 1.68 million possible combinations can be generated, providing ample scalability within each subclass. In cases in which lipid structures are obtained from other sources such as LipidBank or LIPIDAT, the corre-

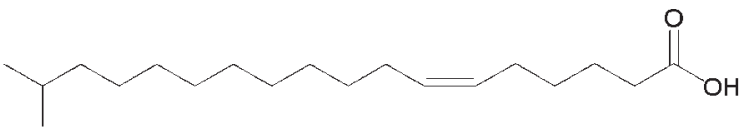

(b) Methyl branched fatty acids: 17-methyl-6Z-octadecenoic acid

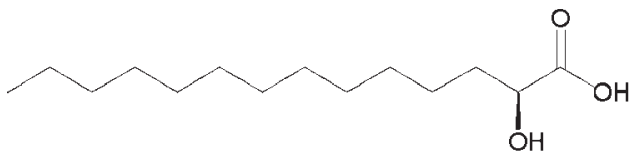

(d) Hydroxy fatty acids: 2S-hydroxy-tetradecanoic acid

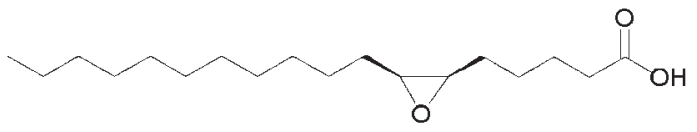

(f) Epoxy fatty acids: 6R,7S-epoxy-octadecanoic acid

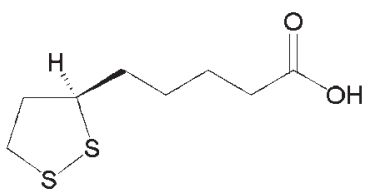

(h) Thia fatty acids: R-Lipoic acid; 1,2-dithiolane-3R-pentanoic acid

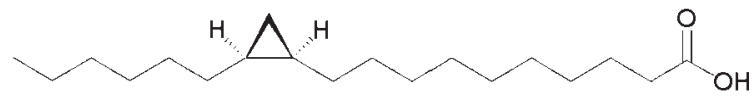

(j) Carbocyclic fatty acids: lactobacillic acid; 11R,12S-methyleneoctadecanoic acid

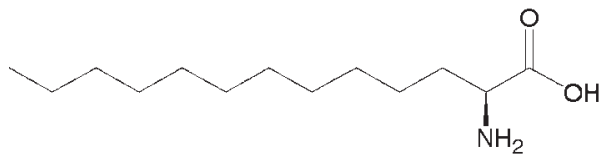

(j) Amino fatty acids: 2S-amino-tridecanoic acid<smiles>CCCC/C(Br)=C/C(=O)O</smiles>

(n) Halogenated fatty acids: 3-bromo-2Z-heptenoic acid sponding IDs for those databases will be included to enable cross-referencing. The first two characters of the ID contain the database identifier (e.g., LM for LIPID MAPS), although other databases may choose to use their own two character identifier (at present, LB for Lipid Bank and LD for LIPIDAT) and assign the last four or more characters uniquely while retaining characters 3 to 8 , which pertain to classification. The corresponding IDs of the other data- 
<smiles>CCCCC[C@H](O)/C=C/C1C=CC(=O)[C@H]1CCCCCCC(=O)O</smiles>

(p) Prostaglandins: Prostaglandin A1; 15S-hydroxy-9-oxo-10Z,13E-prostadienoic acid<smiles>CCCCC[C@H](O)/C=C/C1O[C@H]2CC[C@@H]1O2</smiles>

(r) Thromboxanes: Thromboxane A2; 9S,11S-epoxy, 15S-hydroxy-thromboxa-5Z,13E-den-1-oic acid

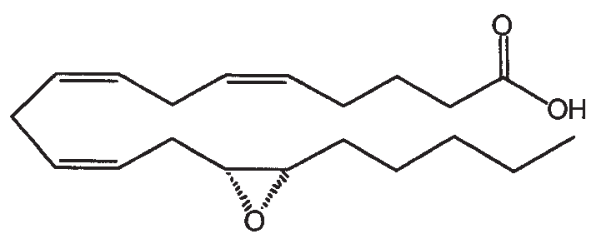

(t) Epoxyeicosatrienoic acids: $14 R, 15 S$ epoxy-5Z,8Z,11Z-eicosarienoic acid<smiles>CCCCC[C@H](O)/C=C/[C@H](C=O)[C@@H](C/C=C/CCCC(=O)O)C(C)=O</smiles>

(v) Levuglandins: LGE2

10,11-seco-9,11-dioxo-15S-hydroxy-5Z,13E-prostadiencic acid<smiles>CCCCC/C=C\CC(O)/C=C/C=C/C=C/C(O)CCCC(=O)O</smiles>

q) Leukdrienes: Leukotriene B4; 5S,12R-dihydroxy-6Z,8E, 10E,14Z-eicosatetraenoic acid<smiles>CCCCC[C@H](O)C(O)/C=C/C=C/C=C\C=C/CCC(=O)O</smiles>
(s) Lipoxins: Lipoxin A4;
5S,6R,15S-tihydroxy-7E,9E, 11Z,13E-eicosatetraenoic acid

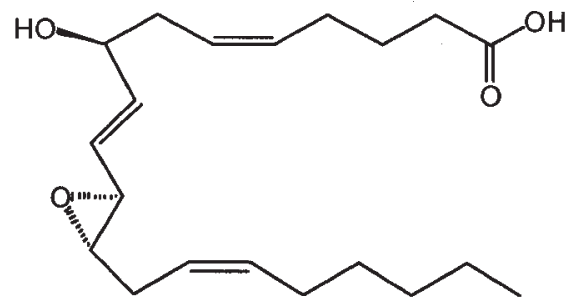

(u) Hepoxilins: Hepoxilin $A 3$ 8R hydoxy-1 1R,12S-epoxy-5Z, GE, 14Z-eicosatrienoic acid

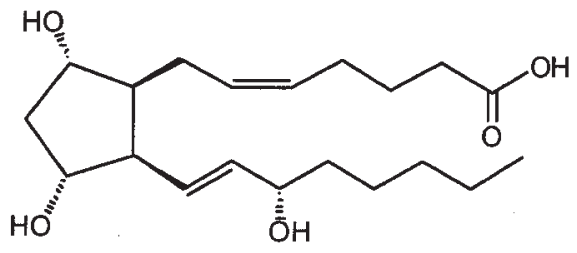

(w) Isoprostanes: $9 S, 11 S, 15 S$-trihydroxy-6Z,13E-prostadienolc acid-cyclo[ $8 \mathrm{~S}, 12 R]$

Fig. 2. Continued.

bases will always be included to enable cross-referencing. Further details regarding the numbering system will be decided by the International Lipids Classification and Nomenclature Committee (see below). In addition to the LIPID ID, each lipid in the database will be searchable by classification (category, class, subclass), systematic name, synonym(s), molecular formula, molecular weight, and many other parameters that are part of its ontology. An important feature will be the databasing of molecular structures, allowing the user to perform web-based substructure searches and structure retrieval across the database. This aim will be accomplished with a chemistry cartridge software component that will enable structures in formats such as MDL molfile and Chemdraw CDX to be imported directly into Oracle database tables.
Furthermore, many lipids, in particular the glycerolipids, glycerophospholipids, and sphingolipids, may be conveniently described in terms of a shorthand name in which abbreviations are used to define backbones, head groups, and sugar units and the radyl substituents are defined by a descriptor indicating carbon chain length and number of double bonds. These shorthand names lend themselves to fast, efficient text-based searches and are used widely in lipid research as compact alternatives to systematic names. The glycerophospholipids in the LIPIDAT database, for example, may be conveniently searched with a shorthand notation that has been extended to handle side chains with acyl, ether, branched-chain, and other functional groups (7). We propose the use of a shorthand notation for selected lipid categories (Table 3) that incorporates a 


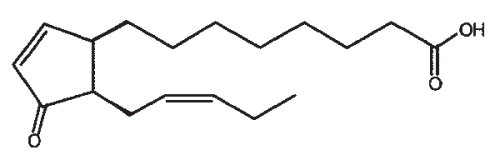

(x) Octadecanoids: 12-oxophytodienoic acid metabol ites: $(9 R, 13 R)-12-0 \times 0$-phyto-10Z,15Z-dienoic acid

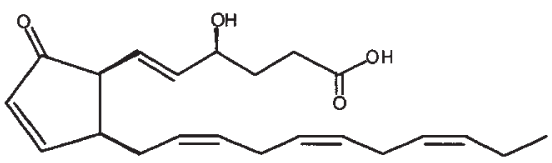

(z) Docosanoids: Neuroprostanes: 4S-hydroxy-8-oxo$(5 \mathrm{E}, 9 \mathrm{Z}, 13 \mathrm{Z}, 16 \mathrm{Z}, 19 \mathrm{Z})$-neuroprostapentaenoic acid-cy clo[7S,11S]

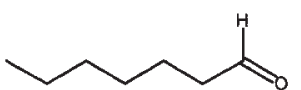

(ab) Fatty aldehydes: heptanal

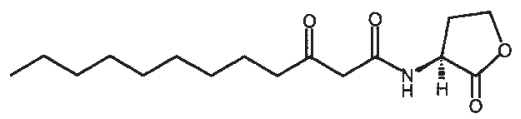

(ad) Fatty amides: Fatty acyl homoserine lactones: $\mathrm{N}$-(3-oxododecanoyl) homoserine lactone

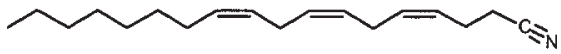

(af) Fatty nitriles: $4 Z, 7 Z, 10 Z$-octadecatrienenitrile

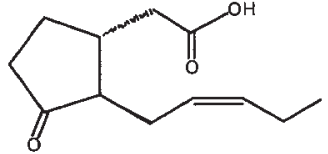

(y) Octadecanoids: Jasmonic acids: jasmonic acid; (1R,2R)-3-oxo-2-(pent 2Z-enyl)-cyclopentaneacetic acid

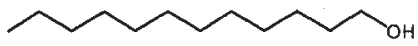

(a) Fatty al cohols: dodecanol

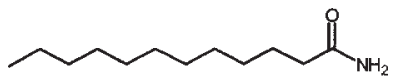

(ac) Fatty amides: $\mathrm{N}$-acyl amides: dodecanamide

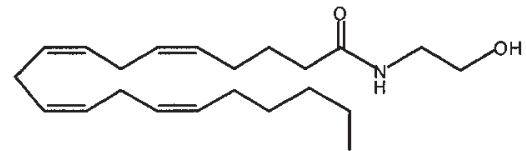

(ae) Fatty amides: $\mathrm{N}$-acyl ethanolamides (endocannabinoids): Anandamide; $\mathrm{N}-(5 \mathrm{Z}, 8 \mathrm{Z}, 11 \mathrm{Z}, 14 \mathrm{Z}$-eicosatetraenoyl)ethanolamine

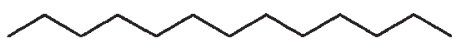

(ag) Hydrocarbons: tridecane

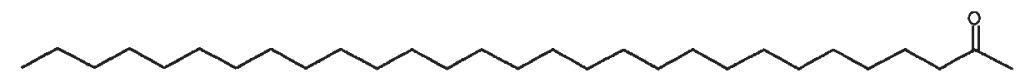

(ah) Oxygenated hydrocarbons: nonacosan-2-one

Fig. 2. Continued.

condensed text nomenclature for glycan substituents. The abbreviations for the sugar units follow the current IUPAC-IUBMB recommendations (4).

\section{LIPID CLASSES AND SUBCLASSES}

\section{Fatty acyls [FA]}

The fatty acyl structure represents the major lipid building block of complex lipids and therefore is one of the most fundamental categories of biological lipids. The fatty acyl group in the fatty acids and conjugates class (Table 4) is characterized by a repeating series of methylene groups that impart hydrophobic character to this category of lipids. The first subclass includes the straight-chain saturated fatty acids containing a terminal carboxylic acid. It could also be considered the most reduced end product of the polyketide pathway. Variants of this structure have one or more methyl substit- uents and encompass quite complex branched-chain fatty acids, such as the mycolic acids. The longest chain in branched-chain fatty acids defines the chain length of these compounds. A considerable number of variations on this basic structure occur in all kingdoms of life (9-12), including fatty acids with one or more double bonds and even acetylenic (triple) bonds. Heteroatoms of oxygen, halogen, nitrogen, and sulfur are also linked to the carbon chains in specific subclasses. Cyclic fatty acids containing three to six carbon atoms as well as heterocyclic rings containing oxygen or nitrogen are found in nature. The cyclopentenyl fatty acids are an example of this latter subclass. The thia fatty acid subclass contains sulfur atom(s) in the fatty acid structure and is exemplified by lipoic acid and biotin. Thiols and thioethers are in this class, but the thioesters are placed in the ester class because of the involvement of these and similar esters in fatty acid metabolism and synthesis.

Separate classes for more complex fatty acids with mul- 


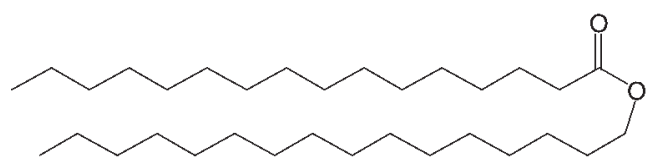

(ai) Wax monoesters: 1-hexadecyl hexadecanoate

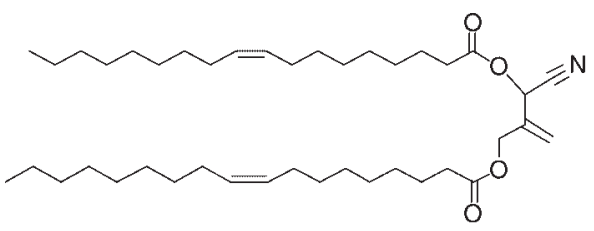

(aj) Cyano esters: 1,3-di-(octadec-9Z-enoyl)-1-cyano-2methylene-propane-1,3-diol<smiles>O=C1CCCCCCCCCCO1</smiles>

(ak) Lactones: 11-undecanolactone

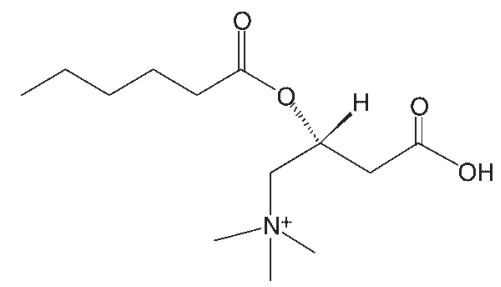

(am) Fatty acyl carnitines: O-hexanoyl-R-carnitine

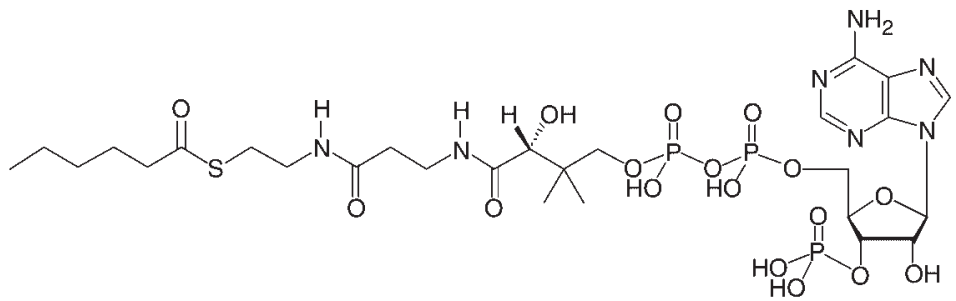

(al) Fatty acyl CoAs: R-hexanoyl CoA

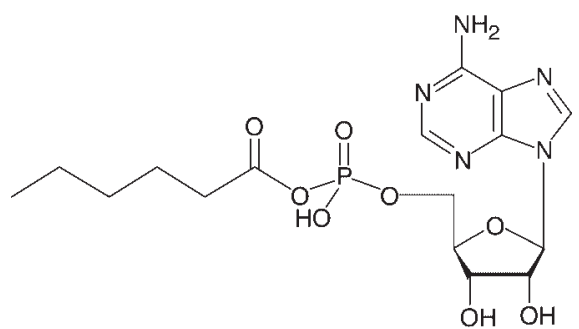

(an) Fatty acyl adenylates: O-hexanoyl-adenosine monophosphate

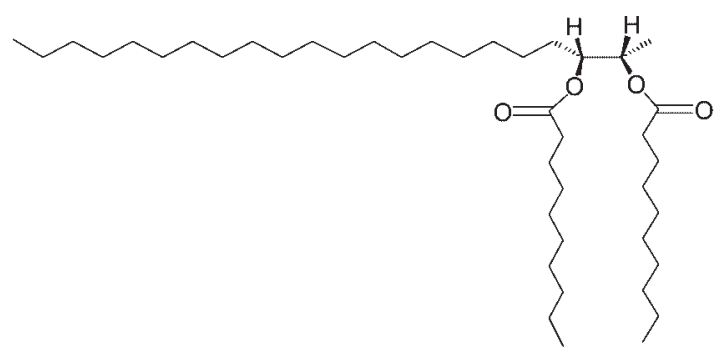

(ao) Wax diesters: 2S,3R-didecanoyl-docosane-2,3-diol

Fig. 2. Continued.

tiple functional groups (but nonbranched) are designated by the total number of carbon atoms found in the critical biosynthetic precursor. These include octadecanoids and lipids in the jasmonic acid pathway of plant hormone biosynthesis, even though jasmonic acids have lost some of their carbon atoms from the biochemical precursor, 12-oxophytodienoic acid (13). Eicosanoids derived from arachidonic acid include prostaglandins, leukotrienes, and other structural derivatives (14). The docosanoids contain 22 carbon atoms and derive from a common precursor, docosahexaenoic acid (15). Many members of these separate subclasses of more complex fatty acids have distinct biological activities.

Other major lipid classes in the fatty acyl category include fatty acid esters such as wax monoesters and diesters and the lactones. The fatty ester class also has subclasses that include important biochemical intermediates such as fatty acyl thioester-CoA derivatives, fatty acyl thioester-acyl carrier protein (ACP) derivatives, fatty acyl carnitines (esters of carnitine), and fatty adenylates, which are mixed anhydrides. The fatty alcohols and fatty aldehydes are typified by terminal hydroxy and oxo groups, respectively. The fatty amides are also $N$-fatty acylated amines and unsubstituted amides, and many simple amides have interesting biological activities in various organisms. Fatty acyl homoserine lactones are fatty amides involved in bacterial quorum sensing (16).

Hydrocarbons are included as a class of fatty acid derivatives because they correspond to six electron reduction products of fatty acids that may have been generated by loss of the carboxylic acid from a fatty acid or fatty acyl moiety during the process of diagenesis in geological samples. Long-chain ethers also have been observed in nature. Chemical structures of the fatty acyls are shown in Fig. 2. 


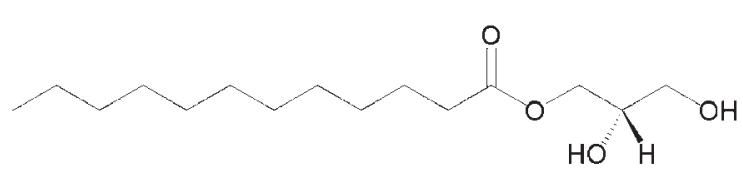

(a) Monoradylgylcerols: Monoacylglycerols: 1-dodecanoyl-sn-glycerol

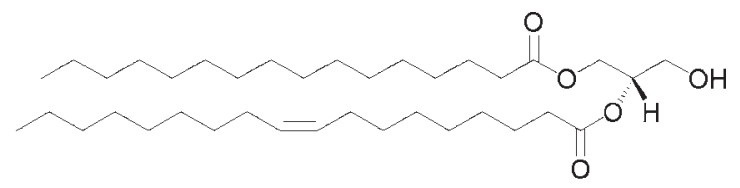

(b) Diradylglycerols: Diacylglycerols 1-hexadecanoyl-2-(9Z-octadecenoyl)-sn-glycerol

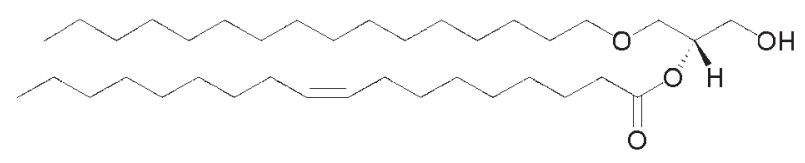

(c) Diradylglycerols: Alkylacylglycerols: 1-O-hexadecyl-2-(9Z-octadecenoyl)-sn-glycerol

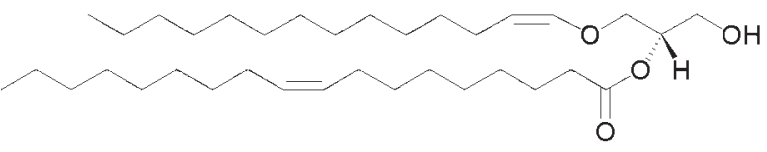

(d) Diradylglycerols: 1Z-alkenylacylglycerols: 1-O-(1Z-tetradecenyl)-2-(9Z-octadecenoyl)-sn-glycerol

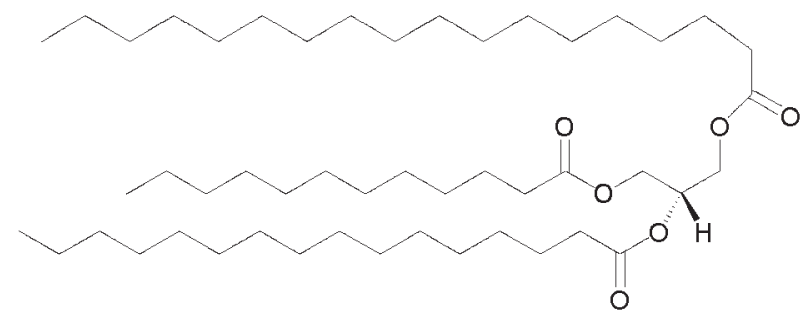

(e) Triradylglycerols: Tricylglycerols:

1-dodecanoyl-2-hexadecanoyl-3-octadecanoyl-sn-glycerol

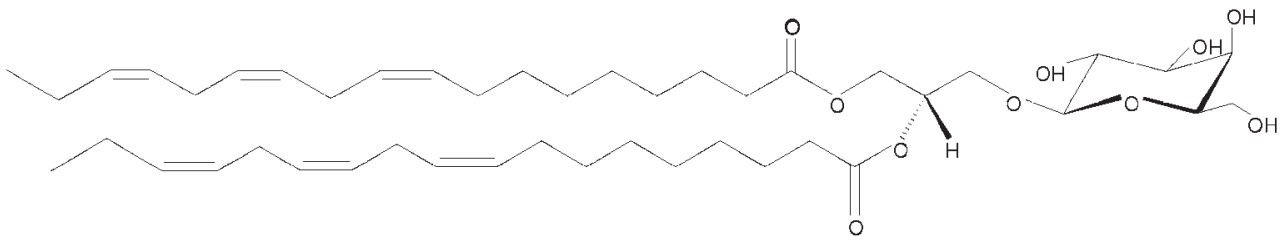

(f) Diradylglycerols: Diacylglycerol glycans:

1,2 di-(9Z,12Z,15Z-octadecatrienoyl)-3-O- $\beta$-D-galactosyl-sn-glycerol

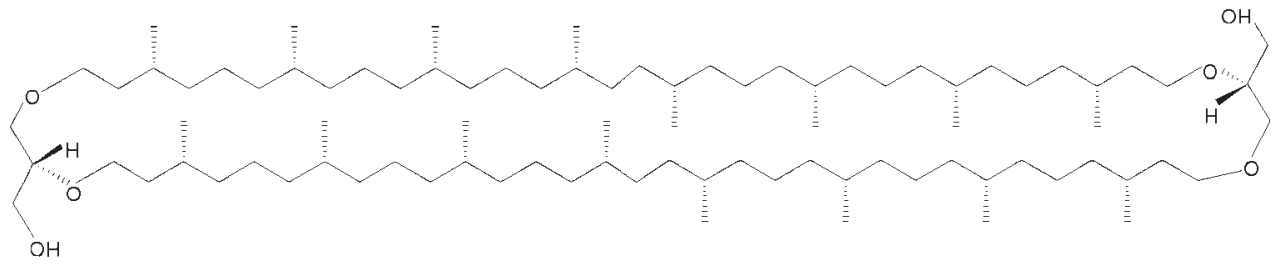

(g) Diradylglycerols: Di-glycerol tetraethers: caldarchaeol

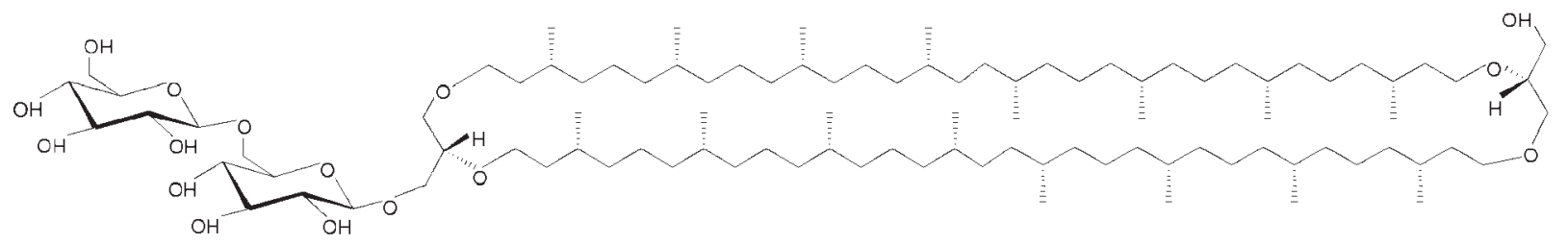

(h) Diradylglycerols: Di-glycerol tetraether glycans: gentiobiosyl-caldarchaeol; Glc $\beta 1$-6Glc $\beta$-caldarchaeol

Fig. 3. Representative structures for glycerolipids.

\section{Glycerolipids [GL]}

The glycerolipids essentially encompass all glycerol-containing lipids. We have purposely made glycerophospholipids a separate category because of their abundance and importance as membrane constituents, metabolic fuels, and signaling molecules. The glycerolipid category (Table 5) is dominated by the mono-, di- and tri-substituted glycerols, the most well-known being the fatty acid esters of glycerol (acylglycerols) $(17,18)$. Additional subclasses are represented by the glycerolglycans, which are character- 
TABLE 6. Glycerophospholipids [GP] classes and subclasses

Glycerophosphocholines [GP01]

Diacylglycerophosphocholines [GP0101]

1-Alkyl,2-acylglycerophosphocholines [GP0102]

1Z-Alkenyl,2-acylglycerophosphocholines [GP0103]

Dialkylglycerophosphocholines [GP0104]

Monoacylglycerophosphocholines [GP0105]

1-Alkyl glycerophosphocholines [GP0106]

1Z-Alkenylglycerophosphocholines [GP0107]

Glycerophosphoethanolamines [GP02]

Diacylglycerophosphoethanolamines [GP0201]

1-Alkyl,2-acylglycerophosphoethanolamines [GP0202]

1Z-Alkenyl,2-acylglycerophosphoethanolamines [GP0203]

Dialkylglycerophosphoethanolamines [GP0204]

Monoacylglycerophosphoethanolamines [GP0205]

1-Alkyl glycerophosphoethanolamines [GP0206]

1Z-Alkenylglycerophosphoethanolamines [GP0207]

Glycerophosphoserines [GP03]

Diacylglycerophosphoserines [GP0301]

1-Alkyl,2-acylglycerophosphoserines [GP0302]

1Z-Alkenyl,2-acylglycerophosphoserines [GP0303]

Dialkylglycerophosphoserines [GP0304]

Monoacylglycerophosphoserines [GP0305]

1-Alkyl glycerophosphoserines [GP0306]

1Z-Alkenylglycerophosphoserines [GP0307]

Glycerophosphoglycerols [GP04]

Diacylglycerophosphoglycerols [GP0401]

1-Alkyl,2-acylglycerophosphoglycerols [GP0402]

1Z-Alkenyl,2-acylglycerophosphoglycerols [GP0403]

Dialkylglycerophosphoglycerols [GP0404]

Monoacylglycerophosphoglycerols [GP0405]

1-Alkyl glycerophosphoglycerols [GP0406]

1Z-Alkenylglycerophosphoglycerols [GP0407]

Diacylglycerophosphodiradylglycerols [GP0408]

Diacylglycerophosphomonoradylglycerols [GP0409]

Monoacylglycerophosphomonoradylglycerols [GP0410]

Glycerophosphoglycerophosphates [GP05]

Diacylglycerophosphoglycerophosphates [GP0501]

1-Alkyl,2-acylglycerophosphoglycerophosphates [GP0502]

1Z-Alkenyl,2-acylglycerophosphoglycerophosphates [GP0503]

Dialkylglycerophosphoglycerophosphates [GP0504]

Monoacylglycerophosphoglycerophosphates [GP0505]

1-Alkyl glycerophosphoglycerophosphates [GP0506]

1Z-Alkenylglycerophosphoglycerophosphates [GP0507]

Glycerophosphoinositols [GP06]

Diacylglycerophosphoinositols [GP0601]

1-Alkyl,2-acylglycerophosphoinositols [GP0602]

1Z-Alkenyl,2-acylglycerophosphoinositols [GP0603]

Dialkylglycerophosphoinositols [GP0604]

Monoacylglycerophosphoinositols [GP0605]

1-Alkyl glycerophosphoinositols [GP0606]

1Z-Alkenylglycerophosphoinositols [GP0607]

Glycerophosphoinositol monophosphates [GP07]

Diacylglycerophosphoinositol monophosphates [GP0701]

1-Alkyl,2-acylglycerophosphoinositol monophosphates [GP0702]

1Z-Alkenyl,2-acylglycerophosphoinositol monophosphates [GP0703]

Dialkylglycerophosphoinositol monophosphates [GP0704]

Monoacylglycerophosphoinositol monophosphates [GP0705]

1-Alkyl glycerophosphoinositol monophosphates [GP0706]

1Z-Alkenylglycerophosphoinositol monophosphates [GP0707]

Glycerophosphoinositol bisphosphates [GP08]

Diacylglycerophosphoinositol bisphosphates [GP0801]

1-Alkyl,2-acylglycerophosphoinositol bisphosphates [GP0802]

1Z-Alkenyl,2-acylglycerophosphoinositol bisphosphates [GP0803]

Monoacylglycerophosphoinositol bisphosphates [GP0804]

1-Alkyl glycerophosphoinositol bisphosphates [GP0805]

1Z-Alkenylglycerophosphoinositol bisphosphates [GP0806]

Glycerophosphoinositol trisphosphates [GP09]

Diacylglycerophosphoinositol trisphosphates [GP0901]

1-Alkyl,2-acylglycerophosphoinositol trisphosphates [GP0902]

1Z-Alkenyl,2-acylglycerophosphoinositol trisphosphates [GP0903]

Monoacylglycerophosphoinositol trisphosphates [GP0904]

1-Alkyl glycerophosphoinositol trisphosphates [GP0905]

1Z-Alkenylglycerophosphoinositol trisphosphates [GP0906]
TABLE 6. (Continued)

Glycerophosphates [GP10]

Diacylglycerophosphates [GP1001]

1-Alkyl,2-acylglycerophosphates [GP1002]

1Z-Alkenyl,2-acylglycerophosphates [GP1003]

Dialkylglycerophosphates [GP1004]

Monoacylglycerophosphates [GP1005]

1-Alkyl glycerophosphates [GP1006]

1Z-Alkenylglycerophosphates [GP1007]

Glyceropyrophosphates [GP11]

Diacylglyceropyrophosphates [GP1101]

Monoacylglyceropyrophosphates [GP1102]

Glycerophosphoglycerophosphoglycerols (cardiolipins) [GP12]

Diacylglycerophosphoglycerophosphodiradylglycerols [GP1201]

Diacylglycerophosphoglycerophosphomonoradylglycerols [GP1202]

1-Alkyl,2-acylglycerophosphoglycerophosphodiradylglycerols [GP1203]

1-Alkyl,2-acylglycerophosphoglycerophosphomonoradylglycerols [GP1204]

1Z-Alkenyl,2-acylglycerophosphoglycerophosphodiradylglycerols [GP1205]

1Z-Alkenyl,2-acylglycerophosphoglycerophosphomonoradylglycerols [GP1206]

Monoacylglycerophosphoglycerophosphomonoradylglycerols [GP1207]

1-Alkyl glycerophosphoglycerophosphodiradylglycerols [GP1208]

1-Alkyl glycerophosphoglycerophosphomonoradylglycerols [GP1209]

1Z-Alkenylglycerophosphoglycerophosphodiradylglycerols [GP1210]

1Z-Alkenylglycerophosphoglycerophosphomonoradylglycerols [GP1211]

CDP-glycerols [GP13]

CDP-diacylglycerols [GP1301]

CDP-1-alkyl,2-acylglycerols [GP1302]

CDP-1 Z-alkenyl,2-acylglycerols [GP1303]

CDP-dialkylglycerols [GP1304]

CDP-monoacylglycerols [GP1305]

CDP-1-alkyl glycerols [GP1306]

CDP-1Z-alkenylglycerols [GP1307]

Glycerophosphoglucose lipids [GP14]

Diacylglycerophosphoglucose lipids [GP1401]

1-Alkyl,2-acylglycerophosphoglucose lipids [GP1402]

1Z-Alkenyl,2-acylglycerophosphoglucose lipids [GP1403]

Monoacylglycerophosphoglucose lipids [GP1404]

1-Alkyl glycerophosphoglucose lipids [GP1405]

1Z-Alkenylglycerophosphoglucose lipids [GP1406]

Glycerophosphoinositolglycans [GP15]

Diacylglycerophosphoinositolglycans [GP1501]

1-Alkyl,2-acylglycerophosphoinositolglycans [GP1502]

1Z-Alkenyl,2-acylglycerophosphoinositolglycans [GP1503]

Monoacylglycerophosphoinositolglycans [GP1504]

1-Alkyl glycerophosphoinositolglycans [GP1505]

1Z-Alkenylglycerophosphoinositolglycans [GP1506]

Glycerophosphonocholines [GP16]

Diacylglycerophosphonocholines [GP1601]

1-Alkyl,2-acylglycerophosphonocholines [GP1602]

1Z-Alkenyl,2-acylglycerophosphonocholines [GP1603]

Dialkylglycerophosphonocholines [GP1604]

Monoacylglycerophosphonocholines [GP1605]

1-Alkyl glycerophosphonocholines [GP1606]

1Z-Alkenylglycerophosphonocholines [GP1607]

Glycerophosphonoethanolamines [GP17]

Diacylglycerophosphonoethanolamines [GP1701]

1-Alkyl,2-acylglycerophosphonoethanolamines [GP1702]

1Z-Alkenyl,2-acylglycerophosphonoethanolamines [GP1703]

Dialkylglycerophosphonoethanolamines [GP1704]

Monoacylglycerophosphonoethanolamines [GP1705]

1-Alkyl glycerophosphonoethanolamines [GP1706]

1Z-Alkenylglycerophosphonoethanolamines [GP1707]

Di-glycerol tetraether phospholipids (caldarchaeols) [GP18]

Glycerol-nonitol tetraether phospholipids [GP19]

Oxidized glycerophospholipids [GP20]

Other [GP00] 


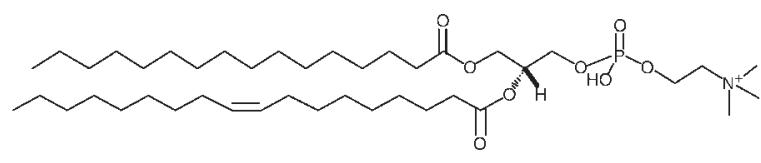

(a) Diacylglycerophosphocholines:

1-hexadecanoyl-2-(9Z-octadecenoyl)-sn-glycero-3-phosphocholine

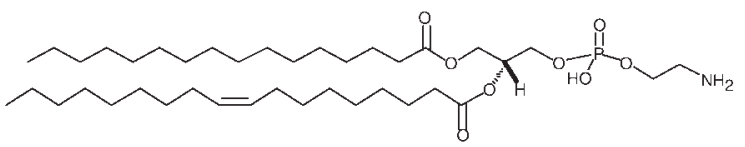

(c) Diacylglycerophosphoethanolamines:

1-hexadecanoyl-2-(9Z-octadecenoyl)-sn-glycero-3-phosphoethanolamine

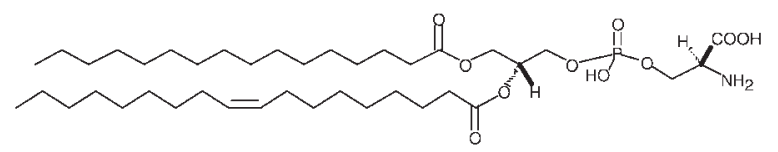

(e) Diacylglycerophosphoserines

1-hexadecanoyl-2-(9Z-octadecenoyl)-sn-glycero-3-phosphoserine

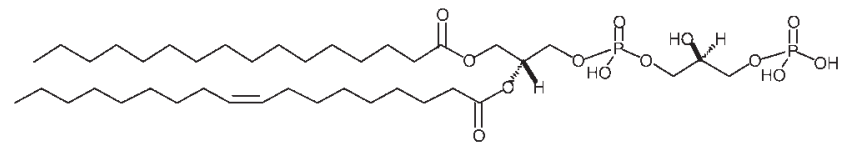

(g) Diacylglycerophosphoglycerophosphates:

1-hexadecanoyl-2-(9Z-octadecenoyl)-sn-glycero-3-phospho-(1'-sn-glycerol-3'-phosphate)

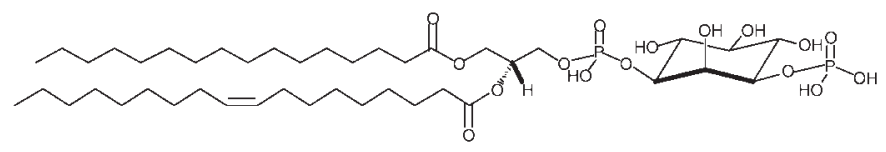

(i) Diacylglycerophosphoinositolmonophosphates:

1-hexadecanoyl-2-(9Z-octadecenoyl)-sn-glycero-3-phospho-(1'-myo-inositol-3'-phosphate)

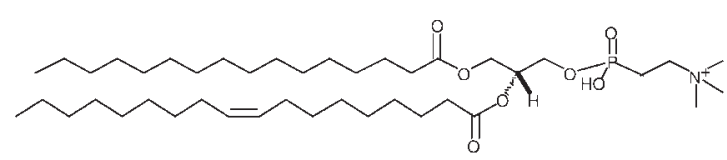

(b) Diacylglycerophosphonocholines:

1-hexadecanoyl-2-(9Z-octadecenoyl)-sn-glycero-3-phosphonocholine

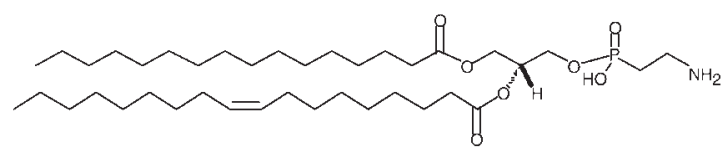

(d) Diacylglycerophosphonoethanolamines:

1-hexadecanoyl-2-(9Z-octadecenoyl)-sn-glycero-3-phosphonoelhanolamine

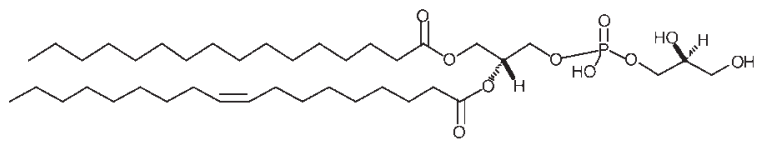

(f) Diacylglycerophosphoglycerols:

1-hexadecanoyl-2-(9Z-octadecenoyl)-sn-glycero-3-phospho-(1'-sn-glycerol)

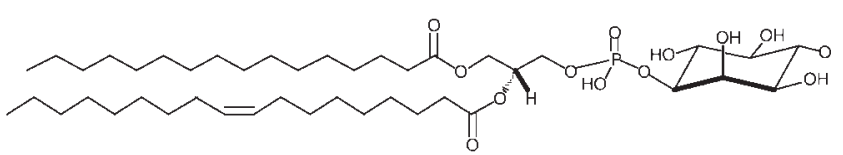

(h) Diacylglycerophosphoinositols:

1-hexadecanoyl-2-(9Z-octadecenoyl)-sn-glycero-3-phospho-(1'-myo-inositol):

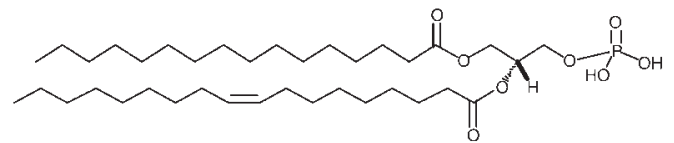

(j) Diacylglycerophosphates (phosphatidic acids):

1-hexadecanoyl-2-(9Z-octadecenoyl)-sn-glycero-3-phosphate

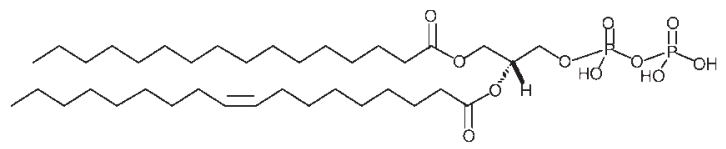

(k) Diacylglyceropyrophosphates:

1-hexadecanoyl-2-(9Z-octadecenoyl)-sn-glycero-3-pyrophosphate

Fig. 4. Representative structures for glycerophospholipids.

ized by the presence of one or more sugar residues attached to glycerol via a glycosidic linkage (19). Examples of structures in this category are shown in Fig. 3. Macrocyclic ether lipids also occur as glycerolipids in the membranes of archaebacteria (20).

\section{Glycerophospholipids [GP]}

The glycerophospholipids are ubiquitous in nature and are key components of the lipid bilayer of cells. Phospholipids may be subdivided into distinct classes (Table 6) based on the nature of the polar "head group" at the $s n-3$ position of the glycerol backbone in eukaryotes and eubacteria or the $s n-1$ position in the case of archaebacteria (21). In the case of the glycerophosphoglycerols and glycerophosphoglycerophosphates, a second glycerol unit constitutes part of the head group, whereas for the glycero- phosphoglycerophosphoglycerols (cardiolipins), a third glycerol unit is typically acylated at the $s n-1$ ' and $s n-2^{\prime}$ positions to create a pseudosymmetrical molecule. Each head group class is further differentiated on the basis of the $s n-1$ and $s n-2$ substituents on the glycerol backbone. Although the glycerol backbone is symmetrical, the second carbon becomes a chiral center when the $s n-1$ and $s n-3$ carbons have different substituents. A large number of trivial names are associated with phospholipids. In the systematic nomenclature, mono/di-radylglycerophospholipids with different acyl or alkyl substituents are designated by similar conventions for naming of classes (see below) and are grouped according to the common polar moieties (i.e., head groups).

Typically, one or both of these hydroxyl groups are acylated with long-chain fatty acids, but there are also alkyl-linked and 1Z-alkenyl-linked (plasmalogen) glycero- 


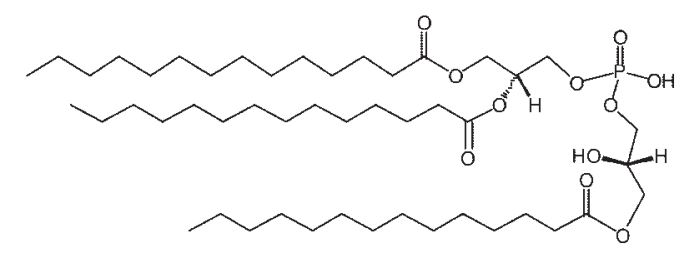

(m) Diacylglycerophosphomonoradylglycerols:

1,2-ditetradecanoyl-sn-glycero-3-phospho-(3'-tetradecanoyl -1'-sn-glycerol)
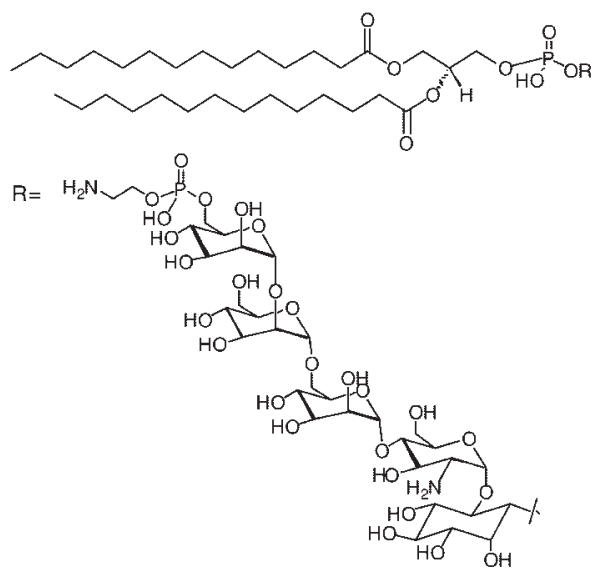

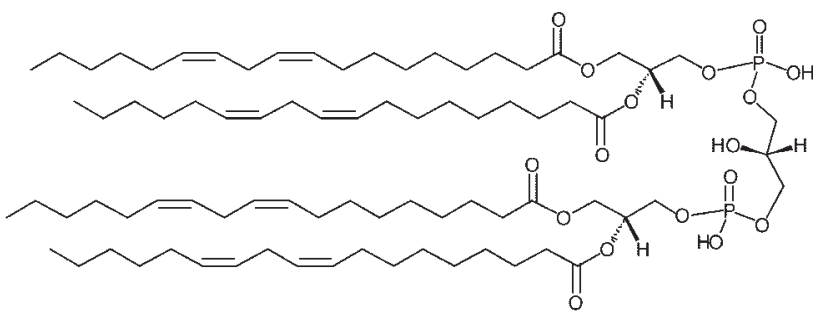

(n) Diacylglycerophosphoglycerophosphodiradylglycerols:

1',3'-Bis[1,2-Di-(9Z-12Z-octadecadienoyl)-sn-glycero-3-phospho]-sn-glycerol

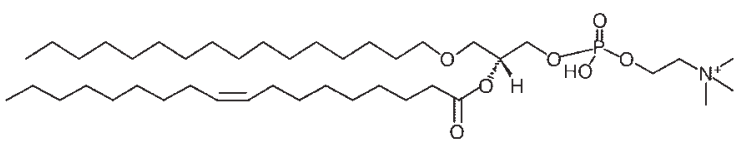

(p) 1-alkyl, 2-acylglycerophosphocholines:

1-O-hexadecyl-2-(9Z-octadecenoyl)-sn-glycero-3-phosphocholine

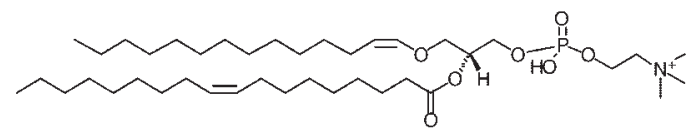

(q) 1Z-alkenyl, 2-acylglycerophosphocholines:

1-O-(1Z-tetradecenyl)-2-(9Z-octadecenoyl)-sn-glycero-3-phosphocholine

(o) Diacylglycerophosphoinositolglycans:

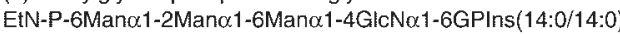

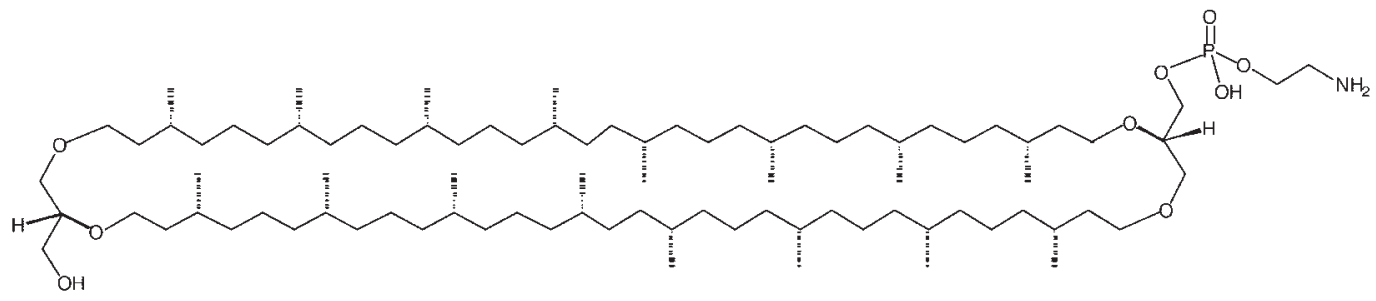

(r) Di-glycerol tetraether phospholipids (caldarchaeols): sn-caldarchaeo-1-phosphoethanolamine

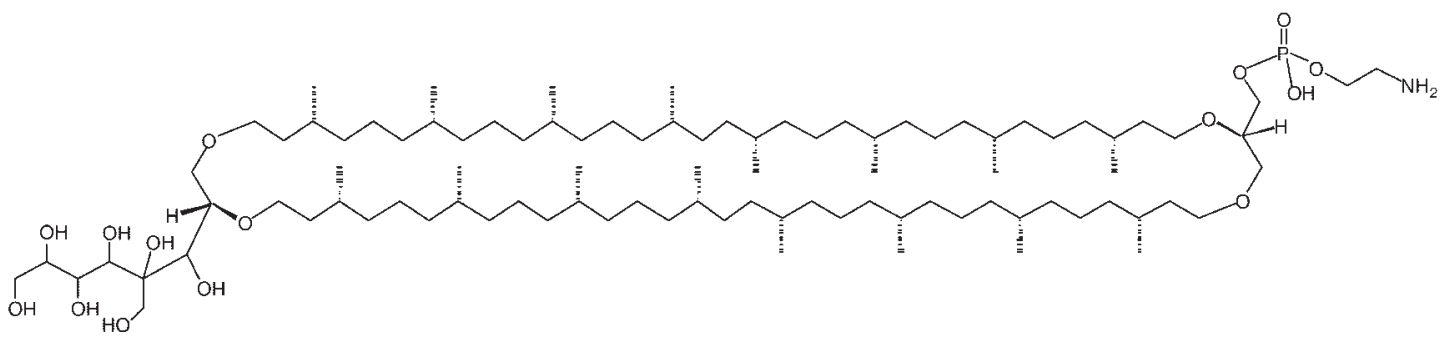

(s) Glycerol-nonitol tetraether phospholipids: s/-caldito-1-phosphoethanolamine

Fig. 4. Continued.

phospholipids, as well as dialkylether variants in prokaryotes. The main biosynthetic pathways for the formation of GPCho and GPEtn (see Table 3 for shorthand notation) were elucidated through the efforts of Kennedy and coworkers (22) in the 1950s and 1960s, and more detailed interconversion pathways to form additional classes of phospholipids were described more recently. In addition to serving as a primary component of cellular membranes and binding sites for intracellular and intercellular proteins, some glycerophospholipids in eukaryotic cells are either precursors of, or are themselves, membrane-derived second messengers. A separate class, called oxidized glycero- phospholipids, is composed of molecules in which one or more of the side chains have been oxidized. Several overviews are available on the classification, nomenclature, metabolism, and profiling of glycerophospholipids (18, 23-26). Structures from this category are shown in Fig. 4.

\section{Sphingolipids [SP]}

Sphingolipids are a complex family of compounds that share a common structural feature, a sphingoid base backbone that is synthesized de novo from serine and a longchain fatty acyl-CoA, then converted into ceramides, phosphosphingolipids, glycosphingolipids, and other species, 


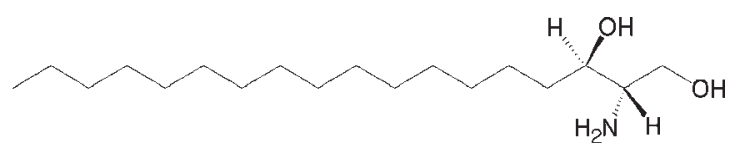

(a) Sphinganines: sphinganine

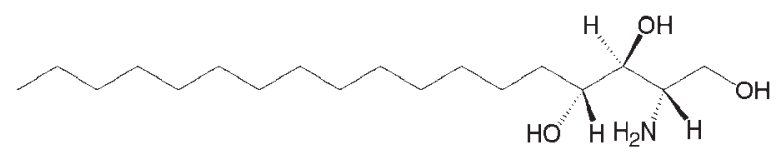

(c) Phytosphingosines: 4-hydroxysphinganine

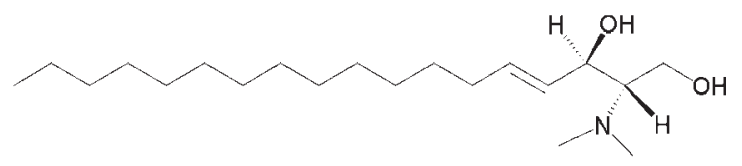

(e) $\mathrm{N}$-methylated sphingoid bases:

$\mathrm{N}, \mathrm{N}$-dimethylsphing-4-enine

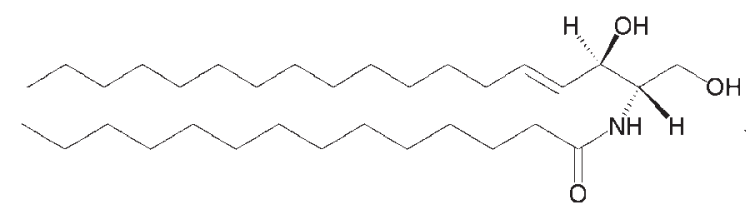

(g) N-acylsphingosines (ceramides):

$\mathrm{N}$-(tetradecanoyl)-sphing-4-enine

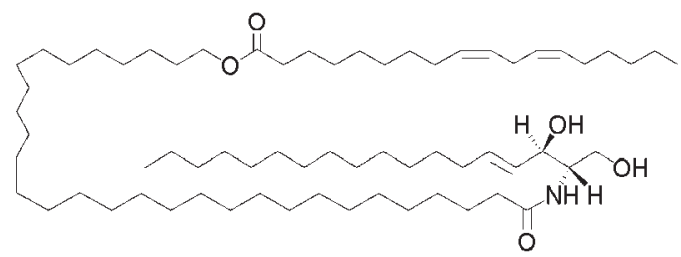

(i) Acylceramides:

$\mathrm{N}-(30-(9 Z, 12 Z$-octadecadienoyloxy)-tricontanoyl)-

sphing-4-enine

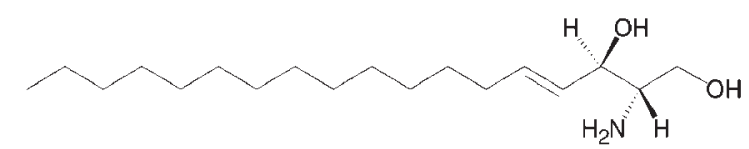

(b) Sphingosines: sphing-4-enine

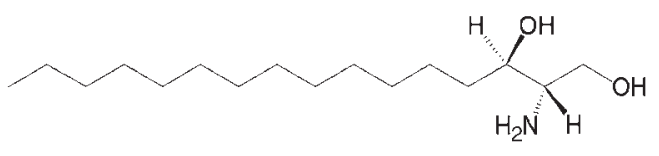

(d) Sphingoid base homologs and variants: hexadecasphinganine

Fig. 5. Representative structures for sphingolipids.

including protein adducts (27, 28). A number of organisms also produce sphingoid base analogs that have many of the same features as sphingolipids (such as long-chain alkyl and vicinal amino and hydroxyl groups) but differ in other features. These have been included in this category because some are known to function as inhibitors or antagonists of sphingolipids, and in some organisms, these types of compounds may serve as surrogates for sphingolipids.

Sphingolipids can be divided into several major classes (Table 7): the sphingoid bases and their simple derivatives (such as the 1-phosphate), the sphingoid bases with an amide-linked fatty acid (e.g., ceramides), and more complex sphingolipids with head groups that are attached via phosphodiester linkages (the phosphosphingolipids), via glycosidic bonds (the simple and complex glycosphingolipids such as cerebrosides and gangliosides), and other groups (such as phosphono- and arseno-sphingolipids). The IUPAC has recommended a systematic nomenclature for sphingolipids (3).

The major sphingoid base of mammals is commonly referred to as "sphingosine," because that name was affixed

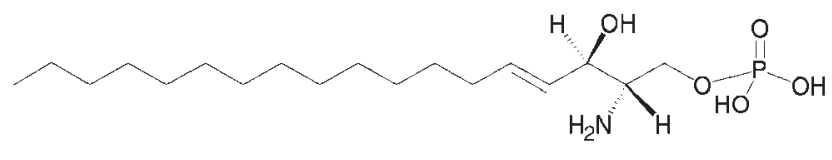

(f) Sphingoid base 1-phosphates: sphing-4-enine-1-phosphate

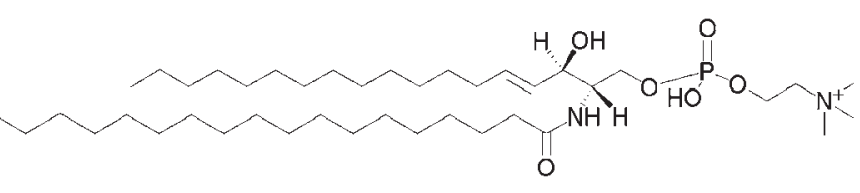

(h) Ceramide phosphocholines (sphingomyelins):

$\mathrm{N}$-(octadecanoyl)-sphing-4-enine-1-phosphocholine

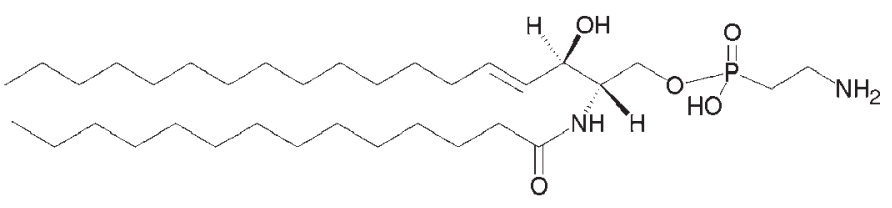

(j) Phosphonosphingolipids:

$\mathrm{N}$-(tetradecanoyl)-sphing-4-enine-1-

(2-aminoethylphosphonate). by the first scientist to isolate this compound (29). Sphingosine is $(2 S, 3 R, 4 E)$-2-aminooctadec-4-ene-1,3-diol (it is also called D-erythro-sphingosine and sphing-4-enine). This is only one of many sphingoid bases found in nature, which vary in alkyl chain length and branching, the number and positions of double bonds, the presence of additional hydroxyl groups, and other features. The structural variation has functional significance; for example, sphingoid bases in the dermis have additional hydroxyls at position 4 (phytoceramides) and/or 6 that can interact with neighboring molecules, thereby strengthening the permeability barrier of skin. Sphingoid bases are found in a variety of derivatives, including the 1-phosphates, lysosphingolipids (such as sphingosine 1-phosphocholine as well as sphingosine 1-glycosides), and $N$-methyl derivatives ( $N$-methyl, $N, N$-dimethyl, and $N, N, N$-trimethyl). In addition, a large number of organisms, such as fungi and sponges, produce compounds with structural similarity to sphingoid bases, some of which (such as myriocin and the fumonisins) are potent inhibitors of enzymes of sphingolipid metabolism. 


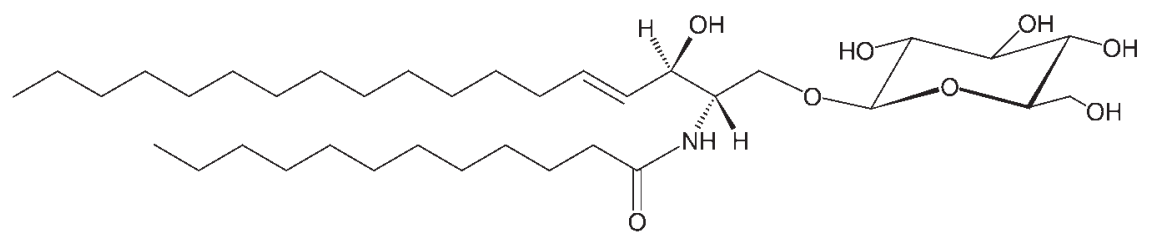

(k) Neutral Glycosphingolipids: Simple Glc series:

Glc $\beta$-Cer(d18:1/12:0)

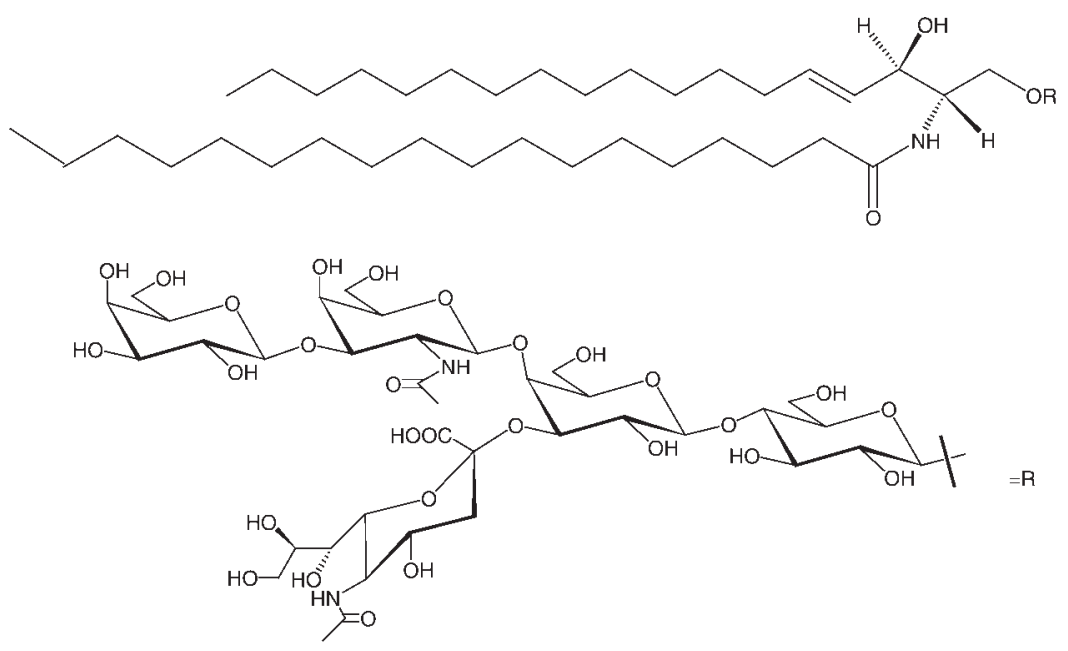

() Acidic Glycosphingolipids: Gangliosides:

Gal $\beta 1-3 G a \mid N A c \beta 1-4($ NeuAc $\alpha 2-3)$ Gal $\beta 1-4$ Glc $\beta$-Cer(d18:1/18:0)

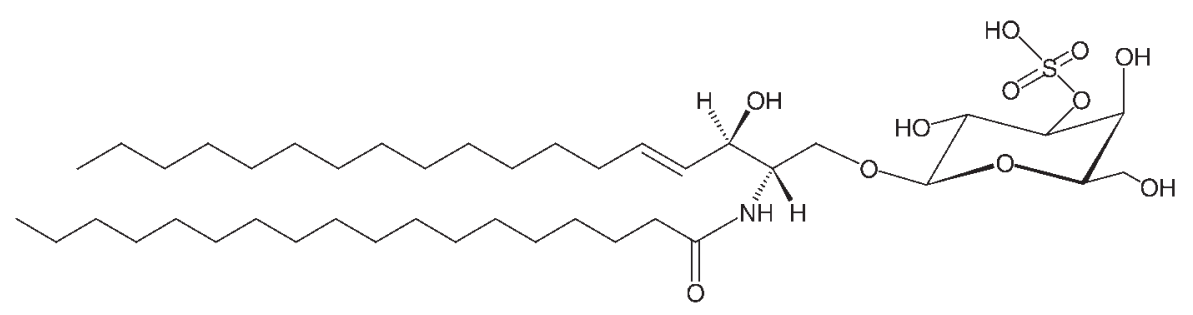

(m) Acidic Glycosphingolipids: Sulfosphingolipids:

(3'-sulfo)Gal $\beta$-Cer(d18:1/18:0)

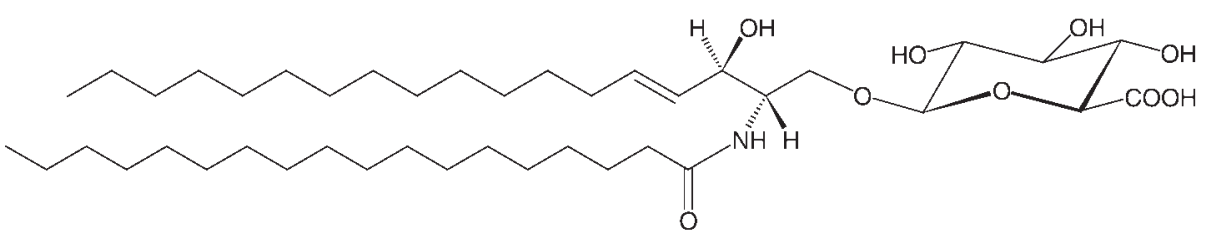

(n) Acidic Glycosphingolipids: Glucuronosphingolipids:

GICUAß-Cer(d18:1/18:0)

Fig. 5. Continued.

Ceramides ( $N$-acyl-sphingoid bases) are a major subclass of sphingoid base derivatives with an amide-linked fatty acid. The fatty acids are typically saturated or monounsaturated with chain lengths from 14 to 26 carbon atoms; the presence of a hydroxyl group on carbon 2 is fairly common. Ceramides sometimes have specialized fatty acids, as illustrated by the skin ceramide in Fig. 5i, which has a 30 carbon fatty acid with a hydroxyl group on the terminal $(\omega)$ carbon. Ceramides are generally precursors of more complex sphingolipids. The major phosphosphingolipids of mammals are sphingomyelins (ceramide phosphocholines), whereas insects contain mainly ceramide phosphoethanolamines and fungi have phytoceramidephosphoinositols and mannose-containing head groups.

Glycosphingolipids (4) are classified on the basis of carbohydrate composition: 1) neutral glycosphingolipids contain one or more uncharged sugars such as glucose (Glu), galactose (Gal), $\mathrm{N}$-acetylglucosamine (GlcNAc), $\mathrm{N}$-acetylga- 
TABLE 7 . Sphingolipids [SP] classes and subclasses

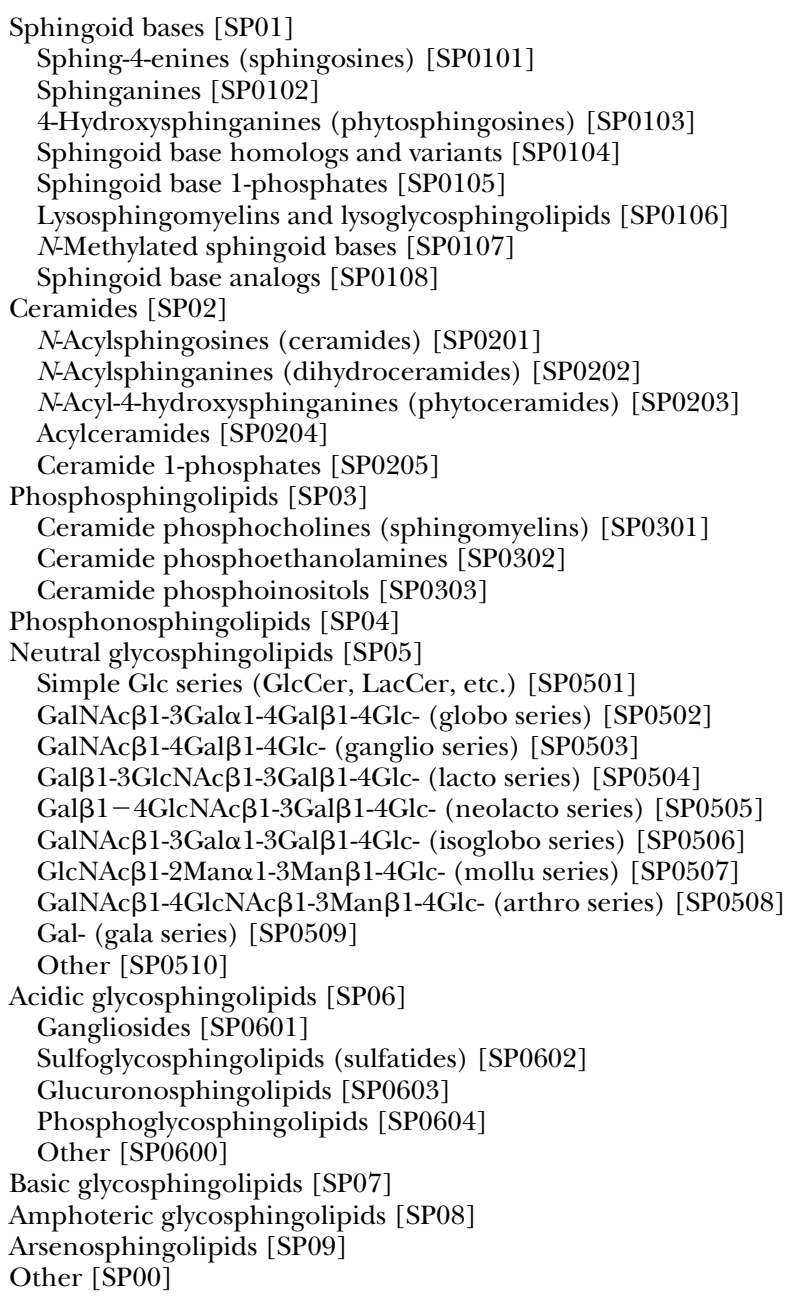

lactosamine (GalNAc), and fucose (Fuc), which are grouped into families based on the nature of the glyco- substituents as shown in the listing; 2) acidic glycosphingolipids contain ionized functional groups (phosphate or sulfate) attached to neutral sugars or charged sugar residues such as sialic acid ( $N$-acetyl or $N$-glycoloyl neuraminic acid). The latter are called gangliosides, and the number of sialic acid residues is usually denoted with a subscript letter (i.e., mono-, di- or tri-) plus a number reflecting the subspecies within that category; 3) basic glycosphingolipids; 4) amphoteric glycosphingolipids. For a few glycosphingolipids, historically assigned names as antigens and blood group structures are still in common use (e.g., Lewis $\mathrm{x}$ and sialyl Lewis $\mathrm{x}$ ). Some aquatic organisms contain sphingolipids in which the phosphate is replaced by a phosphono or arsenate group. The other category includes sphingolipids that are covalently attached to proteins; for example, $\omega$-hydroxyceramides and $\omega$-glucosylceramides are attached to surface proteins of skin, and inositol-phosphoceramides are used as membrane anchors for some fungal proteins in a manner analogous to the glycosylphosphatidylinositol anchors that are attached to proteins in other eukaryotes. Some examples of sphingolipid structures are shown in Fig. 5.
TABLE $8 . \quad$ Sterol lipids [ST] classes and subclasses

Sterols [ST01]

Cholesterol and derivatives [ST0101]

Cholesteryl esters [ST0102]

Phytosterols and derivatives [ST0103]

Marine sterols and derivatives [ST0104]

Fungal sterols and derivatives [ST0105]

Steroids [ST02]

$\mathrm{C}_{18}$ steroids (estrogens) and derivatives [ST0201]

$\mathrm{C}_{19}$ steroids (androgens) and derivatives [ST0202]

$\mathrm{C}_{21}$ steroids (gluco/mineralocorticoids, progestogins) and derivatives [ST0203]

Secosteroids [ST03]

Vitamin $\mathrm{D}_{2}$ and derivatives [ST0301]

Vitamin $\mathrm{D}_{3}$ and derivatives [ST0302]

Bile acids and derivatives [ST04]

$\mathrm{C}_{24}$ bile acids, alcohols, and derivatives [ST0401]

$\mathrm{C}_{26}$ bile acids, alcohols, and derivatives [ST0402]

$\mathrm{C}_{27}$ bile acids, alcohols, and derivatives [ST0403]

$\mathrm{C}_{28}$ bile acids, alcohols, and derivatives [ST0404]

Steroid conjugates [ST05]

Glucuronides [ST0501]

Sulfates [ST0502]

Glycine conjugates [ST0503]

Taurine conjugates [ST0504]

Hopanoids [ST06]

Other [ST00]

\section{Sterol lipids [ST]}

The sterol category is subdivided primarily on the basis of biological function. The sterols, of which cholesterol and its derivatives are the most widely studied in mammalian systems, constitute an important component of membrane lipids, along with the glycerophospholipids and sphingomyelins (30). There are many examples of unique sterols from plant, fungal, and marine sources that are designated as distinct subclasses in this schema (Table 8). The steroids, which also contain the same fused four ring core structure, have different biological roles as hormones

TABLE 9. Prenol lipids [PR] classes and subclasses

\footnotetext{
Isoprenoids [PR01]

$\mathrm{C}_{5}$ isoprenoids [PR0101]

$\mathrm{C}_{10}$ isoprenoids (monoterpenes) [PR0102]

$\mathrm{C}_{15}$ isoprenoids (sesquiterpenes) [PR0103]

$\mathrm{C}_{20}$ isoprenoids (diterpenes) [PR0104]

$\mathrm{C}_{25}$ isoprenoids (sesterterpenes) [PR0105]

$\mathrm{C}_{30}$ isoprenoids (triterpenes) [PR0106]

$\mathrm{C}_{40}$ isoprenoids (tetraterpenes) [PR0107]

Polyterpenes [PR0108]

Quinones and hydroquinones [PR02]

Ubiquinones [PR0201]

Vitamin E [PR0202]

Vitamin K [PR0203]

Polyprenols [PR03]

Bactoprenols [PR0301]

Bactoprenol monophosphates [PR0302]

Bactoprenol diphosphates [PR0303]

Phytoprenols [PR0304]

Phytoprenol monophosphates [PR0305]

Phytoprenol diphosphates [PR0306]

Dolichols [PR0307]

Dolichol monophosphates [PR0308]

Dolichol diphosphates [PR0309]

Other [PR00]
} 


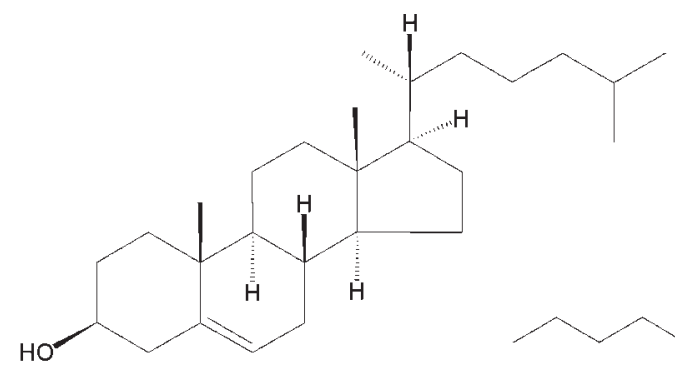

(a) Cholesterol and derivatives: cholesterol; cholest-5-en-3 $\beta$-ol

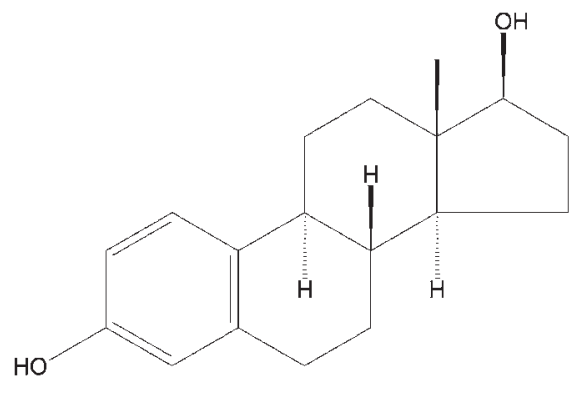

(c) $\mathrm{C}_{18}$ steroids (estrogens) and derivatives: $\beta$-estradiol; 1,3,5[10]-estratriene-3,17/-diol

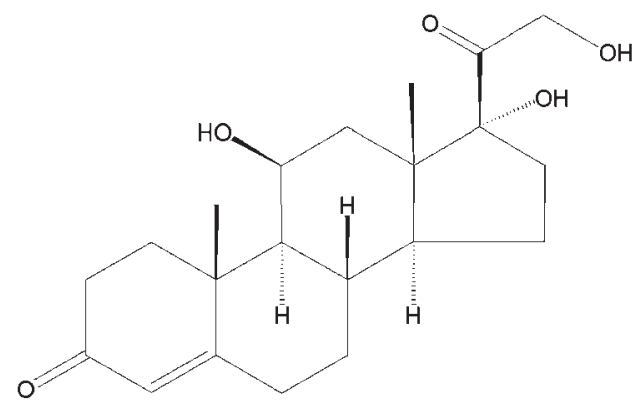

(e) $\mathrm{C}_{21}$ steroids and derivatives:

cortisol; $11 \beta, 17 \alpha, 21$-trihydroxypregn-4-ene-3,20-dione

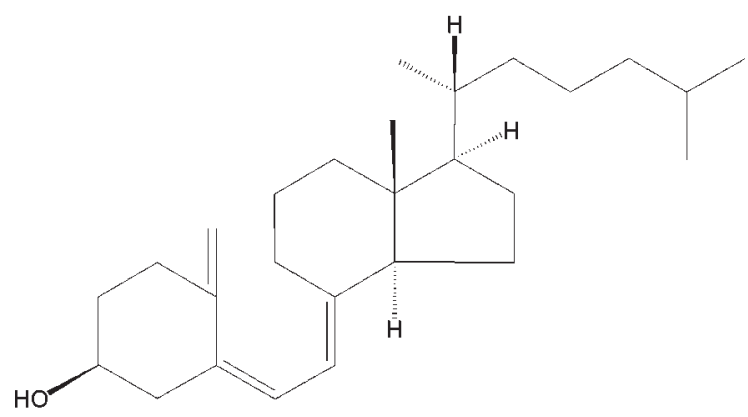

(b) Cholesteryl esters:

cholest-5-en-3 $\beta$-yl dodecanoate

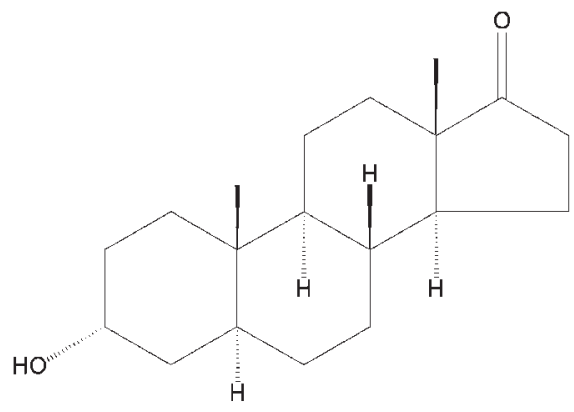

(d) $\mathrm{C}_{19}$ steroids (androgens) and derivatives: androsterone; $3 \alpha$-hydroxy- $5 \alpha$-androstan-17-one

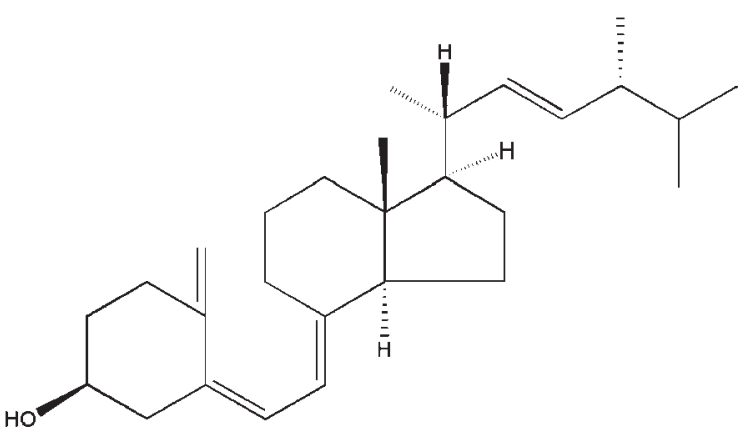

(f) Secosteroids: Vitamin $D_{2}$ and derivatives: vitamin $\mathrm{D}_{2} ;(5 Z, 7 \mathrm{E}, 22 \mathrm{E})-(3 \mathrm{~S})-9,10$-seco-5,7,10(19),22ergostatetraen-3-ol

(g) Secosteroids: Vitamin $D_{3}$ and derivatives: vitamin $D_{3} ;(5 Z, 7 E)-(3 S)-9,10-$ seco-5,7,10(19)-cholestatrien-3-ol

Fig. 6. Representative structures for sterol lipids.

and signaling molecules (31). These are subdivided on the basis of the number of carbons in the core skeleton. The $\mathrm{C}_{18}$ steroids include the estrogen family, whereas the $\mathrm{C}_{19}$ steroids comprise the androgens such as testosterone and androsterone. The $\mathrm{C}_{21}$ subclass, containing a two carbon side chain at the $\mathrm{C}_{17}$ position, includes the pro- gestogens as well as the glucocorticoids and mineralocorticoids. The secosteroids, comprising various forms of vitamin $\mathrm{D}$, are characterized by cleavage of the $\mathrm{B}$ ring of the core structure, hence the "seco" prefix (32). Additional classes within the sterols category are the bile acids (33), which in mammals are primarily derivatives of cholan- 


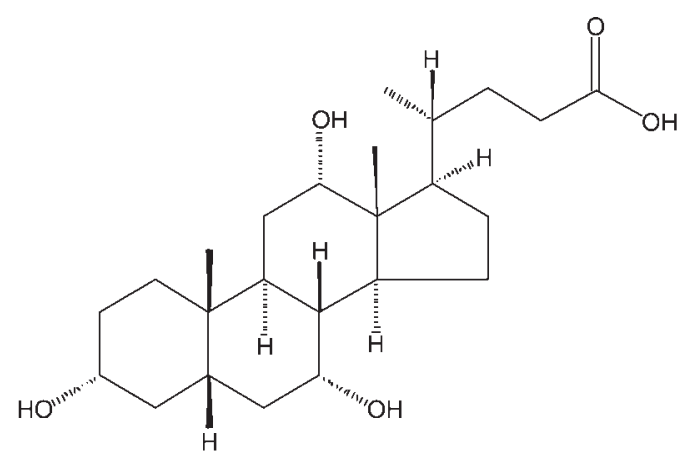

(h) $\mathrm{C}_{24}$ bile acids, alcohols, and derivatives:

cholic acid; $3 \alpha, 7 \alpha, 12 \alpha$-trihydroxy-5 $\beta$-cholan-24-oic acid

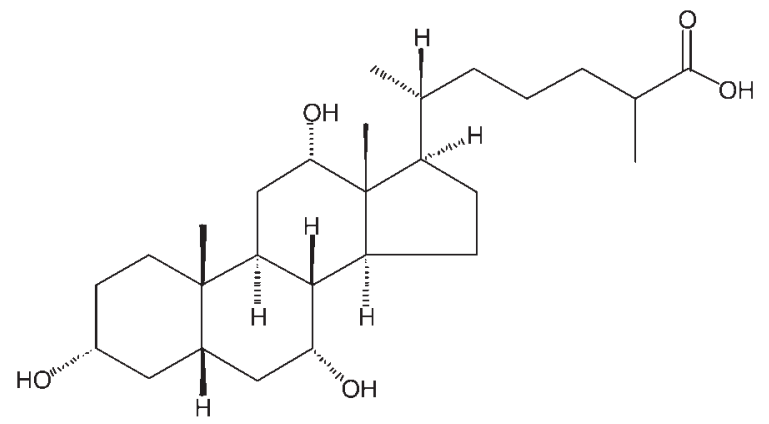

(j) $\mathrm{C}_{27}$ bile acids, alcohols, and derivatives:

$3 \alpha, 7 \alpha, 12 \alpha$-trihydroxy-5 $\beta$-cholestan-26-oic acid

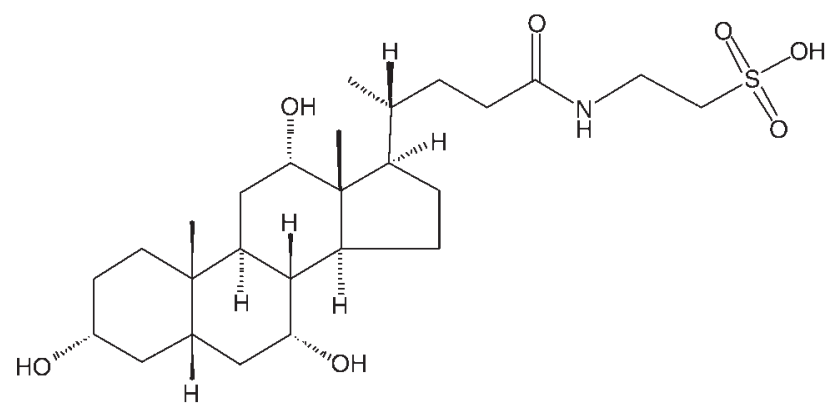

(l) Steroid conjugates: Taurine conjugates: taurocholic acid;

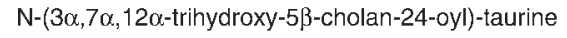

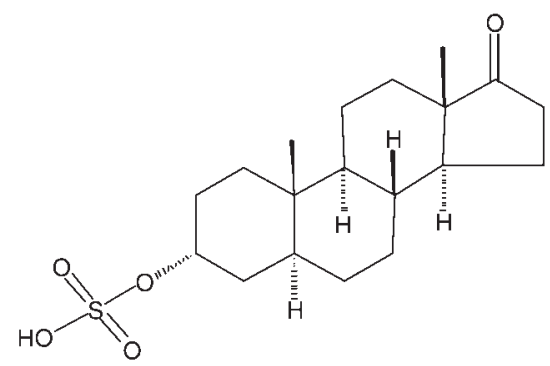

(n) Steroid conjugates: Sulfates: $5 \alpha$-androstane-3 $\alpha$-ol-17-one sulfate

Fig. 6. Continued.

24-oic acid synthesized from cholesterol in the liver and their conjugates (sulfuric acid, taurine, glycine, glucuronic acid, and others). Sterol lipid structures are shown in Fig. 6.

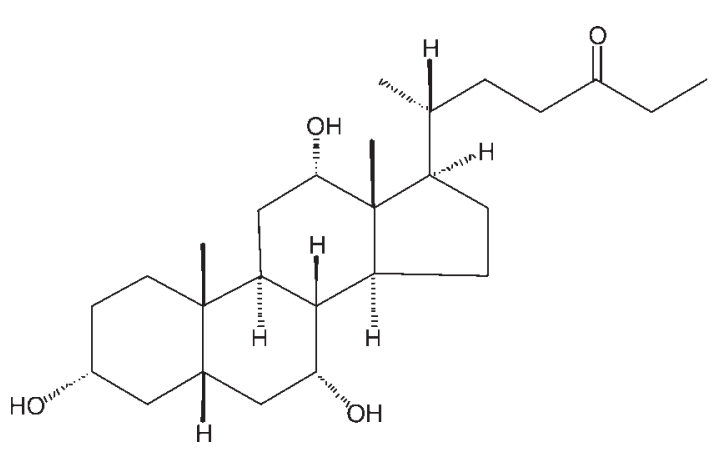

(i) $\mathrm{C}_{26}$ bile acids, alcohols, and derivatives:

$3 \alpha, 7 \alpha, 12 \alpha$-trihydroxy-27-nor-5 $\beta$-cholestan-24-one

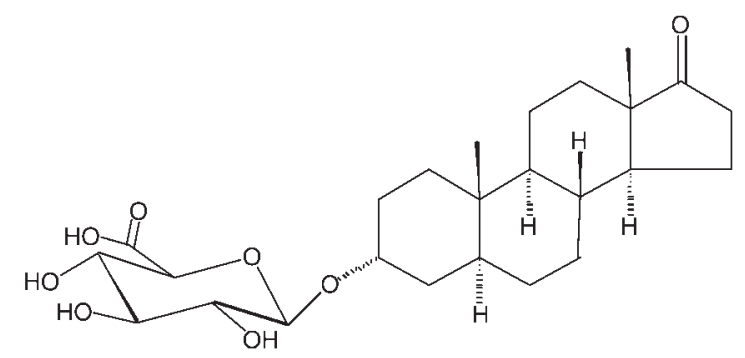

(k) Steroid conjugates: Glucuronides:

$5 \alpha$-androstane-3 $\alpha$-ol-17-one glucuronide

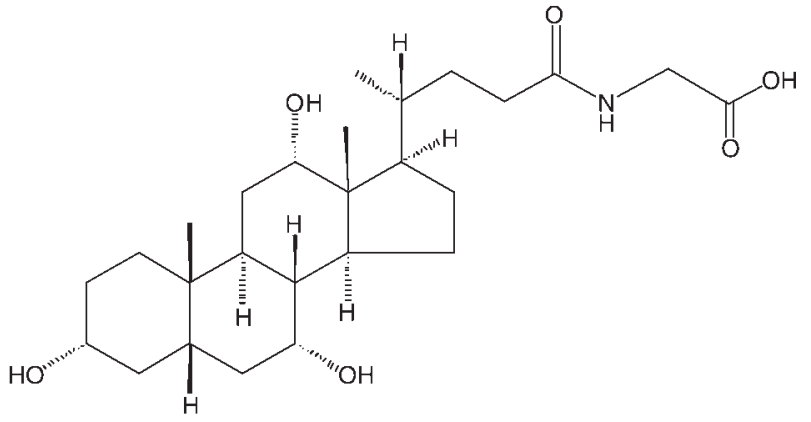

(m) Steroid conjugates: Glycine conjugates: glycocholic acid; $\mathrm{N}-(3 \alpha, 7 \alpha, 12 \alpha$-trihydroxy-5 $\beta$ cholan-24-oyl)-glycine

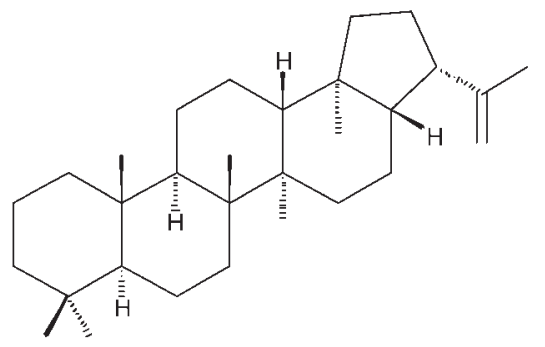

(o) Hopanoids: diploptene; hop-22(29)-ene

\section{Prenol lipids [PR]}

Prenols are synthesized from the five carbon precursors isopentenyl diphosphate and dimethylallyl diphosphate that are produced mainly via the mevalonic acid pathway 


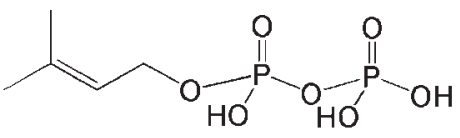

(a) $\mathrm{C}_{5}$ isoprenoids:dimethylallyl pyrophosphate; 3-methylbut-2-enyl pyrophosphate

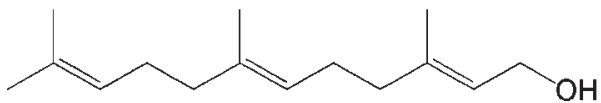

(c) $\mathrm{C}_{15}$ isoprenoids: $2 \mathrm{E}, 6 \mathrm{E}$-farnesol

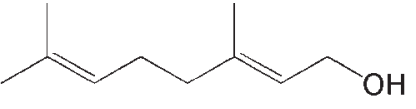

(b) $\mathrm{C}_{10}$ isoprenoids: $2 \mathrm{E}$-geraniol

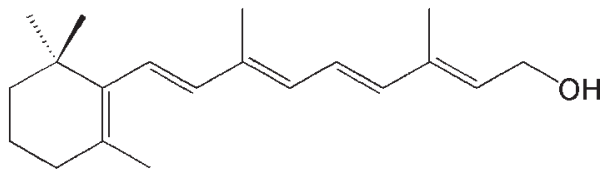

(d) $\mathrm{C}_{20}$ isoprenoids: retinol; vitamin $\mathrm{A}$

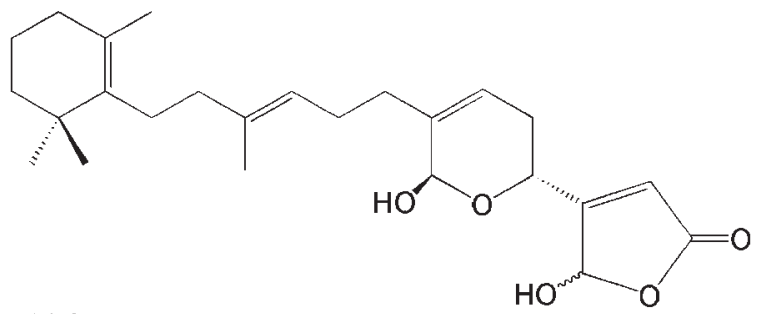

(e) $\mathrm{C}_{25}$ isoprenoids: manoalide
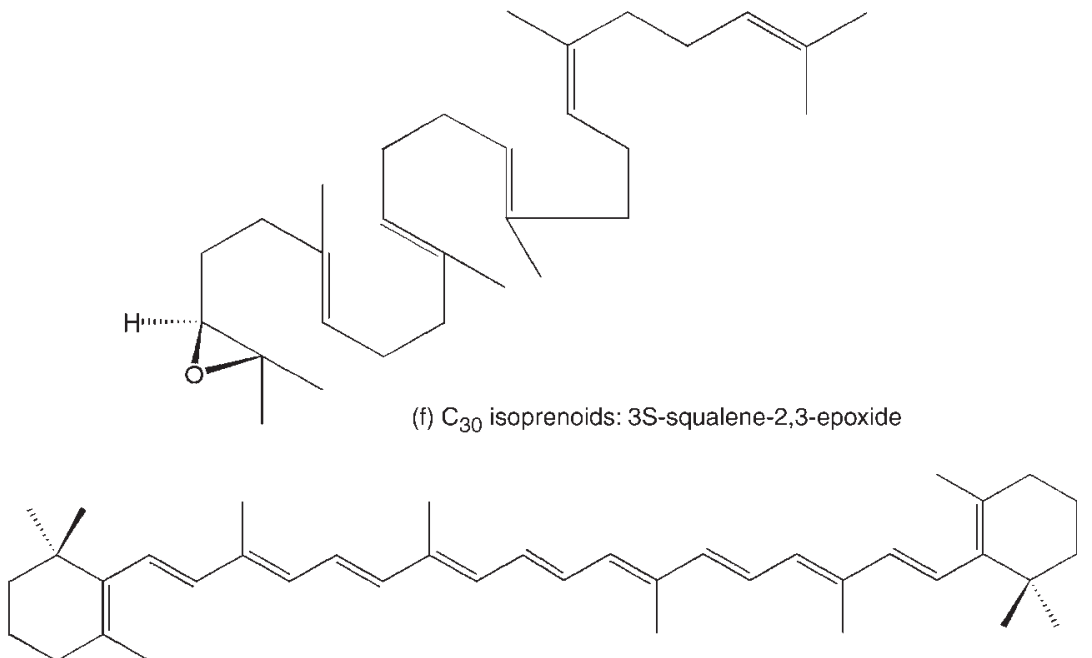

(g) $\mathrm{C}_{40}$ isoprenoids: $\beta$-carotene

Fig. 7. Representative structures for prenol lipids.

(34). In some bacteria (e.g., Escherichia coli) and plants, isoprenoid precursors are made by the methylerythritol phosphate pathway (35). Because the simple isoprenoids (linear alcohols, diphosphates, etc.) are formed by the successive addition of $\mathrm{C}_{5}$ units, it is convenient to classify them in this manner (Table 9), with a polyterpene subclass for those structures containing more than 40 carbons (i.e., $>8$ isoprenoid units) (36). Note that vitamin A and its derivatives and phytanic acid and its oxidation product pristanic acid are grouped under $\mathrm{C}_{20}$ isoprenoids. Carotenoids are important simple isoprenoids that function as antioxidants and as precursors of vitamin A (37). Another biologically important class of molecules is exemplified by the quinones and hydroquinones, which contain an isoprenoid tail attached to a quinonoid core of nonisoprenoid origin. Vitamins E and $\mathrm{K}(38,39)$ as well as the ubiquinones (40) are examples of this class.
Polyprenols and their phosphorylated derivatives play important roles in the transport of oligosaccharides across membranes. Polyprenol phosphate sugars and polyprenol diphosphate sugars function in extracytoplasmic glycosylation reactions (41), in extracellular polysaccharide biosynthesis [for instance, peptidoglycan polymerization in bacteria (42) ], and in eukaryotic protein $N$-glycosylation $(43,44)$. The biosynthesis and function of polyprenol phosphate sugars differ significantly from those of the polyprenol diphosphate sugars; therefore, we have placed them in separate subclasses. Bacteria synthesize polyprenols (called bactoprenols) in which the terminal isoprenoid unit attached to oxygen remains unsaturated, whereas in animal polyprenols (dolichols) the terminal isoprenoid is reduced. Bacterial polyprenols are typically 10 to 12 units long (40), whereas dolichols usually consist of 18 to 22 isoprene units. In the phytoprenols of plants, the three distal 
<smiles>COC1=C(OC)C(=O)C(C(C)(C)C=C(C)C)=C(C)C1=O</smiles>

(h) Ubiquinones: ubiquinone-10 (Co-Q10);

2-methyl-3-decaprenyl-5,6-dimethoxy-1,4-benzoquinone

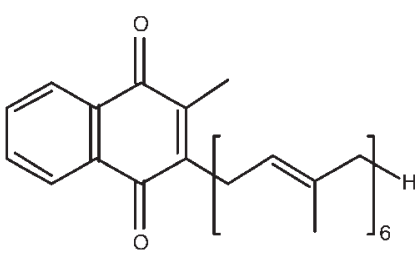

(i) vitamin $\mathrm{K}$ : vitamin $\mathrm{K}_{2}(30)$ :

2-methyl, 3-hexaprenyl-1,4-naphthoquinone; menaquinone-6

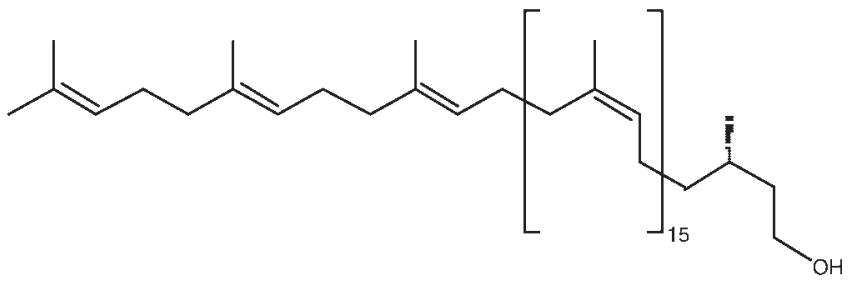

(k) Dolichols: Dol-19; $\alpha$-dihydrononadecaprenol

(j) vitamin $E$ : $\left(2 R, 4^{\prime} R, 8^{\prime} R\right)-\alpha$-tocopherol<smiles>[CH][C@@H](C)CCC[C@H](C)CCCC(C)C</smiles>

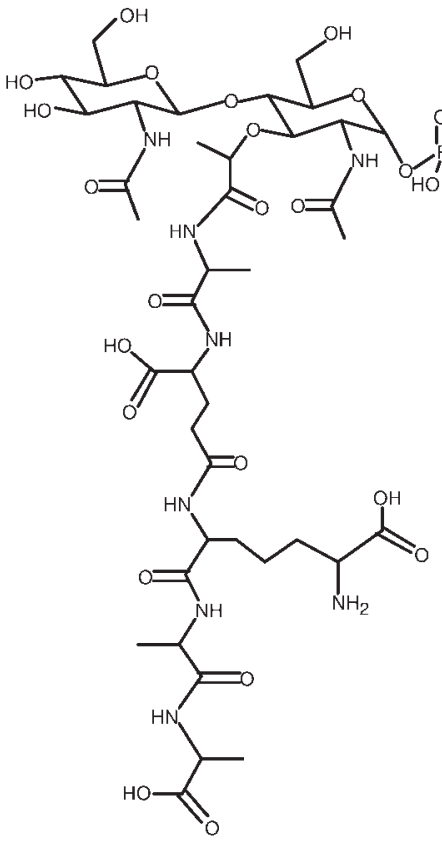

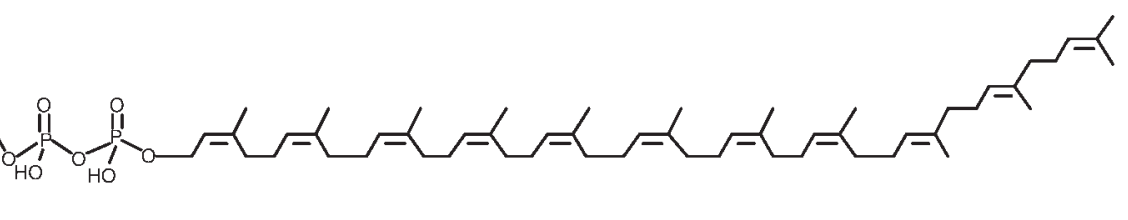

Fig. 7. Continued.

units are reduced. Several examples of prenol lipid structures are shown in Fig. 7.

\section{Saccharolipids [SL]}

We have avoided the term "glycolipid" in the classification scheme to maintain a focus on lipid structures. In fact, all eight lipid categories in the present scheme include important glycan derivatives, making the term glycolipid incompatible with the overall goal of lipid categorization. We have, in addition, coined the term "saccharolipids" to describe compounds in which fatty acids are linked directly to a sugar backbone, forming structures that are compatible with membrane bilayers. In the saccharolipids (Table 10), a sugar substitutes for the glycerol backbone that is present in glycerolipids and glycerophospholipids. Saccharolipids can occur as glycan or as phosphorylated derivatives. The most familiar saccharolipids are the ac- ylated glucosamine precursors of the lipid A component of the lipopolysaccharides in Gram-negative bacteria (41). Typical lipid A molecules are disaccharides of glucosamine, which are derivatized with as many as seven fatty acyl chains $(41,45)$. Note that in naming these compounds, the total number of fatty acyl groups are counted regardless of the nature of the linkage (i.e., amide or ester). The minimal lipopolysaccharide required for growth in E. coli is a hexa-acylated lipid A that is glycosylated with two 3-deoxy-D-mannooctulosonic acid residues (see below). In some bacteria, the glucosamine backbone of lipid A is replaced by 2,3diamino-2,3-dideoxyglucose (46); therefore, the class has been designated "Acylaminosugars." Included also in this class are the Nod factors of nitrogen-fixing bacteria (47), such as Sinorhizobium meliloti. The Nod factors are oligosaccharides of glucosamine that are usually derivatized with a single fatty acyl chain. Additional saccharolipids include 

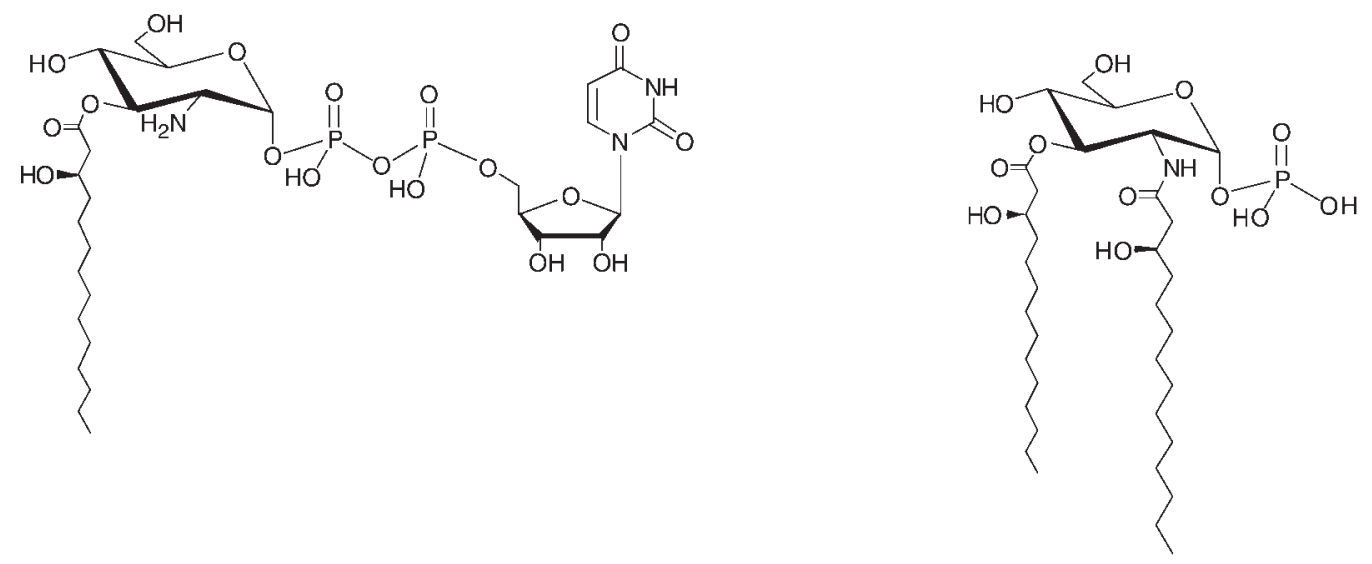

(a) Acylaminosugars: Monoacylaminosugars: UDP-3-O-(3R-hydroxy-tetradecanoyl)-GIcN

(b) Acylaminosugars: Diacylaminosugars: lipid X

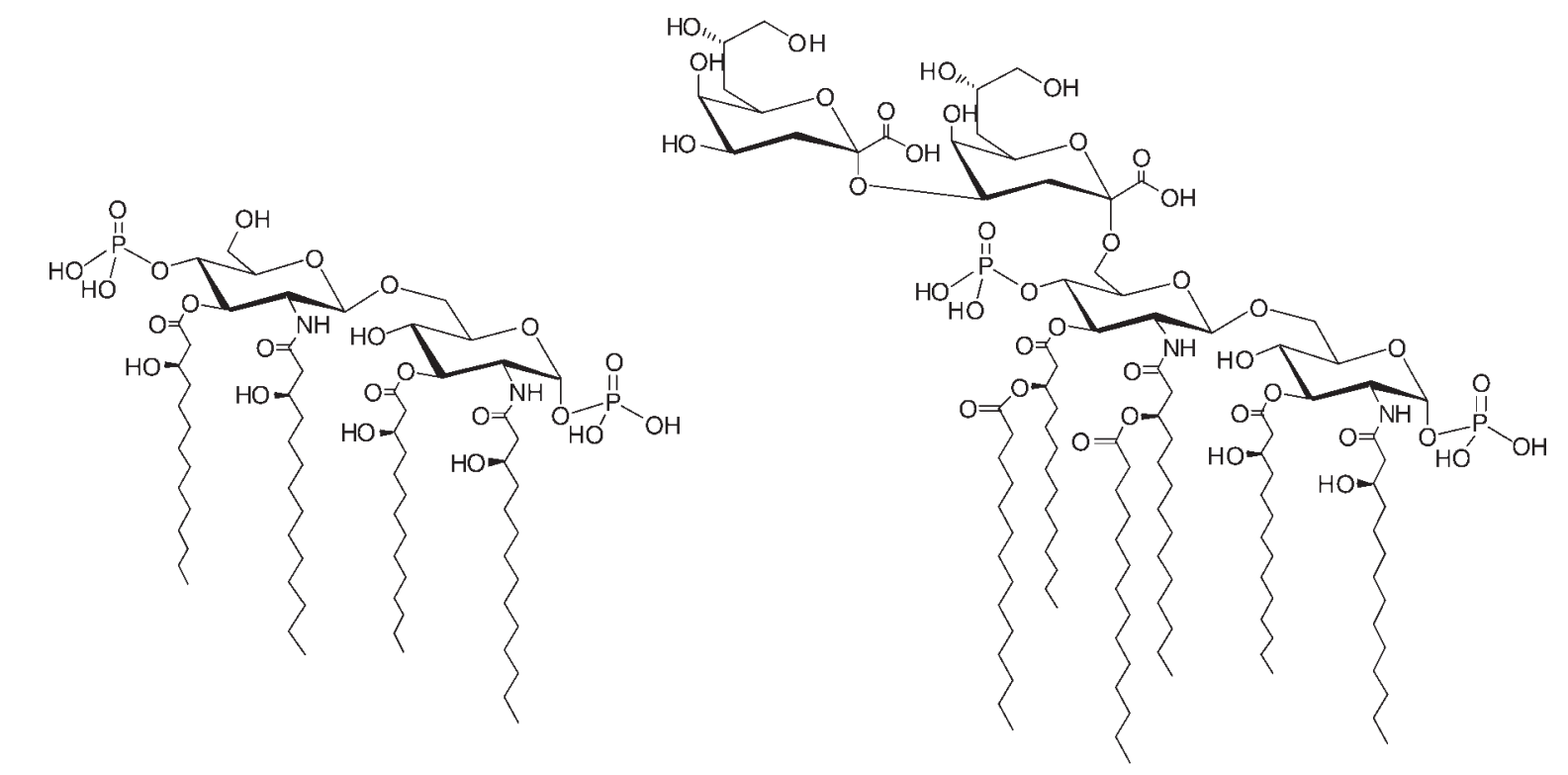

(c) Acylaminosugars: Tetraacylaminosugars: lipid $\mathrm{IV}_{\mathrm{A}}$

(d) Acylaminosugar glycans: $\mathrm{Kdo}_{2}$ lipid A

Fig. 8. Representative structures for saccharolipids.

fatty acylated derivatives of glucose, which are best exemplified by the acylated trehalose units of certain mycobacterial lipids (11). Acylated forms of glucose and sucrose also have been reported in plants (48). Some saccharolipid structures are shown in Fig. 8.

TABLE 10. Saccharolipids [SL] classes and subclasses

\footnotetext{
Acylaminosugars [SL01]

Monoacylaminosugars [SL0101]

Diacylaminosugars [SL0102]

Triacylaminosugars [SL0103]

Tetraacylaminosugars [SL0104]

Pentaacylaminosugars [SL0105]

Hexaacylaminosugars [SL0106]

Heptaacylaminosugars [SL0107]

Acylaminosugar glycans [SL02]

Acyltrehaloses [SL03]

Acyltrehalose glycans [SL04]

Other [SL00]
}

\section{Polyketides [PK]}

Polyketides are synthesized by classic enzymes as well as iterative and multimodular enzymes with semiautonomous active sites that share mechanistic features with the fatty acid synthases, including the involvement of specialized acyl carrier proteins $(49,50)$; however, polyketide synthases generate a much greater diversity of natural product structures, many of which have the character of lipids. The class I polyketide synthases form constrained macrocyclic lactones, typically ranging in size from 14 to 40 atoms, whereas class II and III polyketide synthases generate complex aromatic ring systems (Table 11). Polyketide back-

TABLE 11. Polyketides [PK] classes and subclasses

Macrolide polyketides [PK01]

Aromatic polyketides [PK02]

Nonribosomal peptide/polyketide hybrids [PK03]

Other [PK00] 


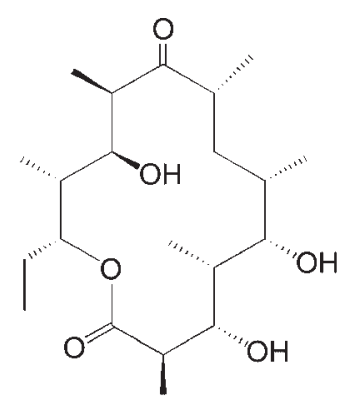

(a) Macrolide polyketides: 6-deoxyerythronolide B

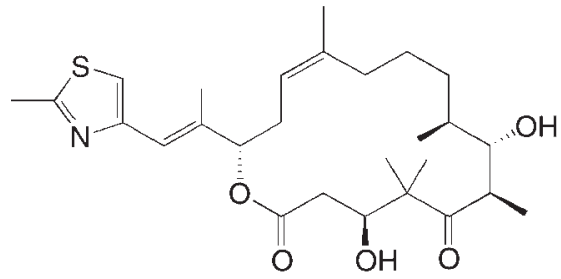

(c) Polyketide hybrids: epothilone D

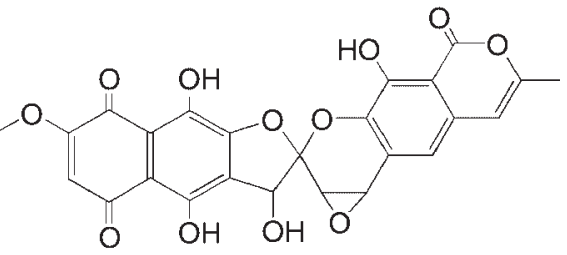

(b) Aromatic polyketides: griseorhodin A

Fig. 9. Representative structures for polyketides.

bones are often further modified by glycosylation, methylation, hydroxylation, oxidation, and/or other processes. Some polyketides are linked with nonribosomally synthesized peptides to form hybrid scaffolds. Examples of the three polyketide classes are shown in Fig. 9. Many commonly used antimicrobial, antiparasitic, and anticancer agents are polyketides or polyketide derivatives. Important examples of these drugs include erythromycins, tetracylines, nystatins, avermectins, and antitumor epothilones. Other polyketides are potent toxins. The possibility of recombining and reengineering the enzymatic modules that assemble polyketides has recently stimulated the search for novel "unnatural" natural products, especially in the antibiotic arena $(51,52)$.

We consider this minimal classification of polyketides as the first step in a more elaborate scheme. It will be important ultimately to include as many polyketide structures as possible in a lipid database that can be searched for substructure and chemical similarity.

\section{DISCUSSION}

The goals of the LIPID MAPS initiative are to characterize known lipids and identify new ones, to quantitate temporal and spatial changes in lipids that occur with cellular metabolism, and to develop bioinformatics approaches that establish dynamic lipid networks; the goals of Lipid Bank (Japan) are to annotate and curate lipid structures and the literature associated with them; and the goals of the European Lipidomics Initiative are to coordinate and organize scientific interactions and workshops associated with lipid research. To coordinate the independent efforts from three continents and to facilitate collaborative work, a comprehensive classification of lipids with a common platform that is compatible with informatics requirements must be developed to deal with the massive amounts of data that will be generated by the lipid community. The proposed classification, nomenclature, and chemical representation system was initially designed to accommodate the massive data that will result from the LIPID MAPS effort, but it has been expanded to accommodate as many lipids as possible. We also have attempted to make the system compatible with existing lipid databases and the lipids currently annotated in them. It is designed to be expandable should new categories, classes, or subclasses be required in the future, and updates will be maintained on the LIPID MAPS website. The development of this system has been enriched by interaction with lipidologists across the world in the hopes that this system will be internationally accepted and used.

The authors appreciate the agreement of the International Lipids Classification and Nomenclature Committee to advise on future issues involving the maintenance of these recommendations. This committee currently includes Edward A. Dennis (chair), Christian Raetz and Robert Murphy representing LIPID MAPS, Friedrich Spener representing the International Conference on the Biosciences of Lipids, Gerrit van Meer representing the European Lipidomics Initiative, and Yousuke Seyama and Takao Shimizu representing the LipidBank of the Japanese Conference on the Biochemistry of Lipids. The authors are most appreciative of informative discussions and encouragement of this effort with Professor Richard Cammack, King's College, London, who is the Chairman of the Nomenclature Committee of IUBMB and the IUPAC/IUBMB Joint Commission on Biochemical Nomenclature. The authors thank the Consortium for Functional Glycomics (headed by Ram Sasisekharan at the Massachusetts Institute of Technology) for providing us with their text nomenclature for glycosylated structures. We are grateful to Dr. Jean Chin, Program Director at the National Institutes of General Medical Sciences, for her valuable input to this effort. This work was supported by the LIPID MAPS Large-Scale Collaborative Grant GM-069338 from the National Institutes of Health. 


\section{REFERENCES}

1. Smith, A. 2000. Oxford Dictionary of Biochemistry and Molecular Biology. 2nd edition. Oxford University Press, Oxford, UK.

2. Christie, W. W. 2003. Lipid Analysis. 3rd edition. Oily Press, Bridgewater, UK

3. IUPAC-IUB Commission on Biochemical Nomenclature (CBN). The nomenclature of lipids (recommendations 1976). 1977. Eur. J. Biochem. 79: 11-21; 1977. Hoppe-Seylers Z. Physiol. Chem. 358: 617-631; 1977. Lipids. 12: 455-468; 1977. Mol. Cell. Biochem. 17: 157-171; 1978. Chem. Phys. Lipids. 21: 159-173; 1978. J. Lipid Res. 19: 114-128; 1978. Biochem. J. 171: 21-35 (http://www.chem.qmul. ac.uk/iupac/lipid/)

4. IUPAC-IUB Joint Commission on Biochemical Nomenclature (JCBN). Nomenclature of glycolipids (recommendations 1997). 2000. Adv. Carbohydr. Chem. Biochem. 55: 311-326; 1988. Carbohydr. Res. 312: 167175; 1998. Eur. J. Biochem. 257: 293-298; 1999. Glycoconjugate J. 16: 1-6; 1999. J. Mol. Biol. 286: 963-970; 1997. Pure Appl. Chem. 69: 2475-2487 (http://www.chem.qmul.ac.uk/iupac/misc/glylp.html).

5. IUPAC-IUB Joint Commission on Biochemical Nomenclature (JCBN). 1987. Nomenclature of prenols (recommendations 1987). Eur. J. Biochem. 167: 181-184 (http://www.chem.qmul.ac.uk/iupac/ $\mathrm{misc} /$ prenol.html).

6. IUPAC-IUB Joint Commission on Biochemical Nomenclature (JCBN). 1989. Nomenclature of steroids (recommendations 1989). Eur. J. Biochem. 186: 429-458 (http://www.chem.qmul.ac.uk/iupac/steroid/).

7. Caffrey, M., and J. Hogan. 1992. LIPIDAT: a database of lipid phase transition temperatures and enthalpy changes. Chem. Phys. Lipids. 61: 1-109 (http://www.lipidat.chemistry.ohio-state.edu).

8. Watanabe, K., E. Yasugi, and M. Ohshima. 2000. How to search the glycolipid data in "Lipidbank for web," the newly-developed lipid database in Japan. Trends Gycosci. Glycotechnol. 12: 175-184.

9. Vance, D. E., and J. E. Vance, editors. 2002. Biochemistry of Lipids, Lipoproteins and Membranes. 4th edition. Elsevier Science, New York.

10. Small, D. M. 1986. The Physical Chemistry of Lipids. Handbook of Lipid Research. Vol. 4. D. J. Hanahan, editor. Plenum Press, New York.

11. Brennan, P. J., and H. Nikaido. 1995. The envelope of mycobacteria. Annu. Rev. Biochem. 64: 29-63.

12. Ohlrogge, J. B. 1997. Regulation of fatty acid synthesis. Annu. Rev. Plant Physiol. Plant Mol. Biol. 48: 109-136.

13. Agrawal, G. K., S. Tamogami, O. Han, H. Iwahasi, and R. Rakwal. 2004. Rice octadecanoid pathway. Biochem. Biophys. Res. Commun. 317: $1-15$.

14. Murphy, R. C., and W. L. Smith. 2002. The eicosanoids: cyclooxygenase, lipoxygenase, and epoxygenase pathways. In Biochemistry of Lipids, Lipoproteins and Membranes. 4th edition. D. E. Vance and J. E. Vance, editors. Elsevier Science, New York. 341-371.

15. Bazan, N. G. 1989. The metabolism of omega-3 polyunsaturated fatty acids in the eye: the possible role of docosahexaenoic acid and docosanoids in retinal physiology and ocular pathology. Prog. Clin. Biol. Res. 312: 95-112.

16. Roche, D. M., J. T. Byers, D. S. Smith, F. G. Glansdorp, D. R. Spring, and M. Welch. 2004. Communications blackout? Do N-acylhomoserine-lactone-degrading enzymes have any role in quorum sensing? Microbiology. 150: 2023-2028.

17. Stam, H., K. Schoonderwoerd, and W. C. Hulsmann. 1987. Synthesis, storage and degradation of myocardial triglycerides. Basic Res. Cardiol. 82 (Suppl. 1): 19-28.

18. Coleman, R. A., and D. P. Lee. 2004. Enzymes of triacylglycerol synthesis and their regulation. Prog. Lipid Res. 43: 134-176.

19. Pahlsson, P., S. L. Spitalnik, P. F. Spitalnik, J. Fantini, O. Rakotonirainy, S. Ghardashkhani, J. Lindberg, P. Konradsson, and G. Larson. 1998. Characterization of galactosyl glycerolipids in the HT29 human colon carcinoma cell line. Arch. Biochem. Biophys. 396: 187198.

20. Koga, Y., M. Nishihara, H. Morii, and M. Akagawa-Matsushita. 1983. Ether polar lipids of methanogenic bacteria: structures, comparative aspects and biosyntheses. Microbiol. Rev. 57: 164-182.

21. Pereto, J., P. Lopez-Garcia, and D. Moreira. 2004. Ancestral lipid biosynthesis and early membrane evolution. Trends Biochem. Sci. 29: 469-477.

22. Kennedy, E. P. 1962. The metabolism and function of complex lipids. Harvey Lecture Series. 57: 143-171.

23. G. Cevc, editor. 1993. Phospholipids Handbook. Marcel Dekker Inc., New York.

24. Forrester, J. S., S. B. Milne, P. T. Ivanova, and H. A. Brown. 2004.
Computational lipidomics: a multiplexed analysis of dynamic changes in membrane lipid composition during signal transduction. Mol. Pharmacol. 65: 813-821.

25. Ivanova, P. T., S. B. Milne, J. S. Forrester, and H. A. Brown. 2004 Lipid arrays: new tools in the understanding of membrane dynamics and lipid signaling. Mol. Interventions. 4: 86-96.

26. Cronan, J. E. 2003. Bacterial membrane lipids: where do we stand? Annu. Rev. Microbiol. 57: 203-224.

27. Merrill, A. H., Jr., and K. Sandhoff. 2002. Sphingolipids: metabolism and cell signaling. In New Comprehensive Biochemistry: Biochemistry of Lipids, Lipoproteins, and Membranes. D. E. Vance and J. E. Vance, editors. Elsevier Science, New York. 373-407.

28. Taniguchi, N., K. Honke, and M. Fukuda. 2002. Handbook of Glycosyltransferases and Related Genes. Springer-Verlag, Tokyo.

29. Thudichum, J. L. W. 1884. A Treatise on the Chemical Constitution of Brain. Bailliere, Tindall, and Cox, London.

30. Bach, D., and E. Wachtel. 2003. Phospholipid/cholesterol model membranes: formation of cholesterol crystallites. Biochim. Biophys. Acta. 1610: 187-197.

31. Tsai, M. J., and B. W. O'Malley. 1994. Molecular mechanisms of action of steroid/thyroid receptor superfamily members. Annu. Rev. Biochem. 63: 451-486.

32. Jones, G., S. A. Strugnell, and H. F. DeLuca. 1998. Current understanding of the molecular actions of vitamin D. Physiol. Rev. 78: 11931231.

33. Russell, D. W. 2003. The enzymes, regulation, and genetics of bile acid synthesis. Annu. Rev. Biochem. 72: 137-174.

34. Kuzuyama, T., and H. Seto. 2003. Diversity of the biosynthesis of the isoprene units. Nat. Prod. Rep. 20: 171-183.

35. Rodriguez-Concepcion, M. 2004. The MEP pathway: a new target for the development of herbicides, antibiotics and antimalarial drugs. Curr. Pharm. Res. 10: 2391-2400.

36. Porter, J. W., and S. L. Spurgeon. 1981. Biosynthesis of Isoprenoid Compounds. Vol. 1. John Wiley \& Sons, New York.

37. Demming-Adams, B., and W. W. Adams. 2002. Antioxidants in photosynthesis and human nutrition. Science. 298: 2149-2153.

38. Ricciarelli, R., J. M. Zingg, and A. AzziI. 2001. Vitamin E: protective role of a Janus molecule. FASEB J. 15: 2314-2325.

39. Meganathan, R. 2001. Biosynthesis of menaquinone (vitamin K2) and ubiquinone (coenzyme $Q$ ): a perspective on enzymatic mechanisms. Vitam. Horm. 61: 173-218.

40. Meganathan, R. 2001. Ubiquinone biosynthesis in microorganisms. FEMS Microbiol. Lett. 203: 131-139.

41. Raetz, C. R. H., and C. Whitfield. 2002. Lipopolysaccharide endotoxins. Annu. Rev. Biochem. 71: 635-700.

42. Lazar, K., and S. Walker. 2002. Substrate analogues to study cell-wall biosynthesis and its inhibition. Curr. Opin. Chem. Biol. 6: 786-793.

43. Schenk, B., F. Fernandez, and C. J. Waechter. 2001. The ins(ide) and out(side) of dolichyl phosphate biosynthesis and recycling in the endoplasmic reticulum. Glycobiology. 11: 61R-71R.

44. Helenius, J., and M. Aebi. 2001. Intracellular functions of N-linked glycans. Science. 291: 2364-2369.

45. Zähringer, U., B. Lindner, and E. T. Rietschel. 1999. Chemical structure of Lipid A: recent advances in structural analysis of biologically active molecules. In Endotoxin in Health and Disease. H. Brade, S. M. Opal, S. N. Vogel, and D. C. Morrison, editors. Marcel Dekker, New York. 93-114.

46. Sweet, C. R., A. A. Ribeiro, and C. R. Raetz. 2004. Oxidation and transamination of the 3'-position of UDP-N-acetylglucosamine by enzymes from Acidithiobacillus ferrooxidans. Role in the formation of lipid A molecules with four amide-linked acyl chains. J. Biol. Chem. 279: 25400-25410.

47. Spaink, H. P. 2000. Root nodulation and infection factors produced by rhizobial bacteria. Annu. Rev. Microbiol. 54: 257-288.

48. Ghangas, G. S., and J. C. Steffens. 1993. UDPglucose: fatty acid transglucosylation and transacylation in triacylglucose biosynthesis. Proc. Natl. Acad. Sci. USA. 90: 9911-9915.

49. Walsh, C. T. 2004. Polyketide and nonribosomal peptide antibiotics: modularity and versatility. Science. 303: 1805-1810.

50. Khosla, C., R. Gokhale, J. R. Jacobsen, and D. E. Cane. 1999. Tolerance and specificity of polyketide synthases. Annu. Rev. Biochem. 68: 219-253

51. Reeves, C. D. 2003. The enzymology of combinatorial biosynthesis. Crit. Rev. Biotechnol. 23: 95-147.

52. Moore, B. S., and C. Hartweck. 2002. Biosynthesis and attachment of novel bacterial polyketide synthase starter units. Nat. Prod. Rep. 19: $70-99$. 